\title{
Systemic Perturbations in Amine and Kynurenine Metabolism Associated with Acute SARS-CoV-2 Infection and Inflammatory Cytokine Responses
}

Nathan G. Lawler, ${ }^{\bigcirc}$ Nicola Gray, ${ }^{๑}$ Torben Kimhofer, Berin Boughton, Melvin Gay, Rongchang Yang, Aude-Claire Morillon, Sung-Tong Chin, Monique Ryan, Sofina Begum, Sze How Bong, Jerome D. Coudert, Dale Edgar, Edward Raby, Sven Pettersson, Toby Richards, Elaine Holmes, Luke Whiley,* and Jeremy K. Nicholson*

Cite This: J. Proteome Res. 2021, 20, 2796-2811

Read Online

ACCESS | Lill Metrics \& More | 回 Article Recommendations | st Supporting Information

ABSTRACT: We performed quantitative metabolic phenotyping of blood plasma in parallel with cytokine/chemokine analysis from participants who were either SARSCoV-2 $(+)(n=10)$ or SARS-CoV-2 $(-)(n=49)$. SARS-CoV-2 positivity was associated with a unique metabolic phenotype and demonstrated a complex systemic response to infection, including severe perturbations in amino acid and kynurenine metabolic pathways. Nine metabolites were elevated in plasma and strongly associated with infection (quinolinic acid, glutamic acid, nicotinic acid, aspartic acid, neopterin, kynurenine, phenylalanine, 3-hydroxykynurenine, and taurine; $p<0.05$ ), while four metabolites were lower in infection (tryptophan, histidine, indole-3-acetic acid, and citrulline; $p<0.05$ ). This signature supports a systemic metabolic phenoconversion following infection, indicating possible neurotoxicity and neurological disruption (elevations of 3-hydroxykynurenine and quinolinic acid) and liver dysfunction

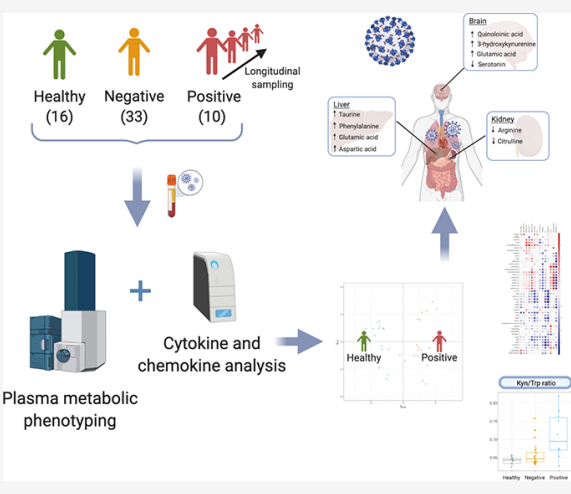
(reduction in Fischer's ratio and elevation of taurine). Finally, we report correlations between the key metabolite changes observed in the disease with concentrations of proinflammatory cytokines and chemokines showing strong immunometabolic disorder in response to SARS-CoV-2 infection.

KEYWORDS: biogenic amines, tryptophan, kynurenine, cytokines, host response, SARS-CoV-2, COVID-19, metabolic phenotyping, phenoconversion

\section{INTRODUCTION}

In the current COVID-19 pandemic, over 118 million people have been diagnosed with severe acute respiratory syndrome coronavirus 2 (SARS-CoV-2) infection and over 2.7 million people have died to date ${ }^{1}$. The manifestation of SARS-CoV-2 infection ranges from asymptomatic to severe respiratory illness, sometimes with a variety of complications. The virus has been reported to cause lymphopenia and systemic hyperinflammatory responses that result in lung, ${ }^{2,3}$ heart, ${ }^{4}$ liver, ${ }^{5,6}$ and kidney damage ${ }^{7}$ and can present in the form of neurological dysfunction, ${ }^{8-10}$ diabetes, ${ }^{1-13}$ and stroke. ${ }^{14}$

While there has been a rapid response from the scientific community resulting in a surge of SARS-CoV-2/COVID-19 focused research, understanding the exact viral pathogenesis, metabolic interactions, and mechanisms that underpin an individual's disease trajectory remains poor. Understanding sequential mechanisms of SARS-CoV-2 action is critical to developing actionable therapeutic interventions to mitigate adverse outcomes following infection. Furthermore, elucidation of the metabolic host responses will enable the stratification of individuals who are poor responders to infection with resulting poor prognosis. Predicting prognosis post-infection will allow for earlier intervention and increase the potential for personalized treatments and improved recovery rates across the population. As with previous human severe acute respiratory syndrome (SARS) and Middle East respiratory syndrome (MERS), there is concern that some of the complex expressions of the disease may have long-term consequences, where the criteria for "recovery" are still ill-defined ${ }^{15}$ and are based mainly on reduction in respiratory symptoms. However, detection of systemic biomarkers related to multiple systems gives the potential for measuring systemic recovery as a well as acute illness.

Received: January 20, 2021

Published: March 16, 2021 
Metabolic phenotyping has proved to be a highly informative approach in systems biology that enriches the understanding of the metabolic mechanisms of infectious disease pathogenesis and can facilitate mechanistic understanding, patient stratification, and prognosis prediction. ${ }^{16,17}$ The approach has shown early promise when applied to SARS-CoV-2 infection, with reports of altered lipid metabolism and perturbations in peripheral amine concentrations in response to infection, ${ }^{18-21}$ with similar findings also reported in previous SARS and MERS coronavirus epidemics. ${ }^{22-24}$

With the rapid global scientific response, studies have shown that individuals post-SARS-CoV-2 infection undergo a metabolic phenoconversion (an altered systemic metabolic state affecting multiple organs in response to antigen) that is indicative of a multi-organ viral impact and may be predictive of infection. $^{21}$ However, there remains a need to further understand the systemic role of altered metabolic states in the human response to SARS-CoV-2 infection, through understanding the metabolic mechanisms and inflammatory relationships involved. To elucidate the systemic effects further, we analyzed 42 blood plasma metabolites from key catabolic pathways known to influence human inflammatory responses. The metabolite panel consisted of amino acids, biogenic amines, and kynurenines. These metabolic data were supplemented with a complementary panel of 14 serum cytokines and chemokines, to characterize the infection and determine the relationship between inflammatory signatures and host immune response.

Analysis was performed on a clinical cohort collected from Western Australia (WA) consisting of four participant groups: (i) SARS-CoV-2 (+), tested positive for SARS-CoV-2 infection via nasopharyngeal swab polymerase chain reaction (PCR) test and were admitted to hospital; (ii) SARS-CoV-2 (-) reported to local testing clinics with COVID-like symptoms but tested negative for the virus and were subsequently sent home; (iii) healthy controls (HC) recruited from the community who reported no signs or symptoms of viral infection and had not been diagnosed with SARS-CoV-2 infection; and (iv) admitted to hospital for treatment for other conditions and tested negative for the virus in routine hospital screening during the outbreak.

Using these four groups, we describe the underlying metabolic signatures and inflammatory relationships that occur in a host response to an acute SARS-CoV-2 infection. Understanding such metabolic signatures of infection is critical to gain mechanistic insights into COVID-19 disease pathogenesis and patient outcomes. Furthermore, the resultant signatures may provide value as putative biomarker panels, which can facilitate patient stratification, augment current clinical diagnosis strategies, and provide clinically actionable information.

\section{METHODS}

\subsection{Patient Enrollment and Sample Collection}

2.1.1. Sample Collection, Perth, Australia. The study was initiated at Fiona Stanley Hospital by the COVID Research Response Collaboration ${ }^{25}$ as part of the International Severe Acute Respiratory and Emerging Infection Consortium (ISARIC)/World Health Organisation (WHO) pandemic trial framework (SMHS Research Governance Office PRN:3976 and Murdoch University Ethics no. 2020/ 052, and no. 2020/053). A full description of the cohort including demographic data (Table $\mathrm{S} 1$ ) is provided in the Supporting Information.

Plasma samples for metabolite analysis and serum samples for cytokine and chemokine analysis were collected from 59 individuals who were (i) SARS-CoV-2 (+): individuals who presented to the hospital reporting symptoms of COVID-19 and, subsequently, tested positive by PCR of nasopharyngeal swab samples $(n=10)$; (ii) SARS-CoV-2 (-): individuals who presented to COVID assessment clinic or hospital emergency department reporting symptoms of COVID-19 and, subsequently, tested negative $(n=23)$; (iii) healthy control (HC): individuals were recruited at a similar time of SARS-CoV-2 $(+)$ patient sample collection $(n=16)$; or (iv) hospitalized individuals receiving care for chronic illnesses who tested negative during routine screening by PCR of nasopharyngeal swab samples $(n=10)$.

\subsection{Quantification of Metabolic Phenotyping Panel}

Samples were not heat-treated prior to analysis (a common technique used in the literature to inactivate viruses in biofluids ${ }^{26}$ ) as heating resulted in substantial alterations in patient metabolite phenotypes. ${ }^{27}$

Measurement of 42 metabolite concentrations was performed using ultrahigh-performance liquid chromatography-mass spectrometry (UHPLC-MS) based on validated assays to provide full quantification for amino acids and the products of tryptophan catabolism. ${ }^{28-30}$ Full details of both assays can be found in the Supporting Information and are described briefly below.

2.2.1. Biogenic Amines and Amino Acids Analysis. The quantification of biogenic amines and amino acids was performed according to methods previously reported. ${ }^{29,30}$ In brief, 27 amino acids were quantified from $10 \mu \mathrm{L}$ of plasma. Sample extraction was completed using a Biomek is sample automation system (Beckman Coulter, Mount Waverley, VIC 3149, Australia). The samples were diluted 1:1 with water, 20 $\mu \mathrm{L}$ of stable isotope-labeled (SIL) internal standards in water was added, and protein precipitation was performed by the addition of $90 \mu \mathrm{L}$ of methanol. After mixing and centrifugation, $10 \mu \mathrm{L}$ of the supernatant was transferred into a Waters $700 \mu \mathrm{L}$ 96-well plate for derivatization with AccQTag reagent (Waters Corp., Milford, MA). The subsequent derivatized samples were diluted 1:4 (v/v) with water prior to liquid chromatography-mass spectrometry (LC-MS) analysis.

LC-MS analysis was performed using a Waters Acquity UPLC (Waters Corp., Milford, MA) coupled to a Bruker impact II QToF-MS (Bruker Daltonics, Bremen, Germany). Full instrument settings are described in the Supporting Information. The resulting raw data files were processed for peak integrations and the calculation of metabolite concentrations using Target Analysis for Screening and Quantification (TASQ) software v2.2 (Bruker Daltonics, Bremen, Germany). Details of additional methods can be found in the Supporting Information.

2.2.2. Tryptophan Metabolic Pathway Analysis. The quantification of tryptophan and 14 catabolites of its metabolic pathway was performed as previously reported. ${ }^{28}$ In brief, tryptophan metabolites were quantified from $50 \mu \mathrm{L}$ of plasma. Sample extraction was completed using a Biomek i5 sample automation system. SIL internal standards $(20 \mu \mathrm{L})$ were added to all samples prior to protein precipitation via the addition of $250 \mu \mathrm{L}$ of methanol containing $2 \mathrm{mM}$ ammonium 


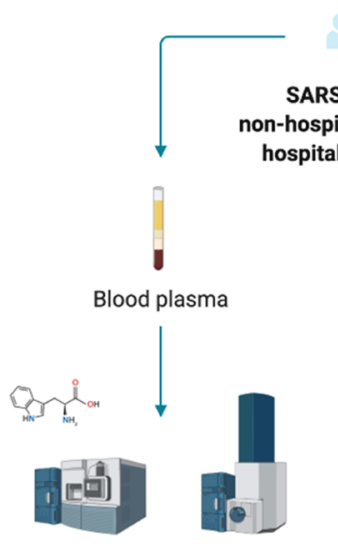

\section{Metabolite quantification performed on all samples for amino acids and tryptophan pathway metabolites. Cytokine and chemokine analysis performed on select healthy control and} SARS-CoV-2 (+) subjects.

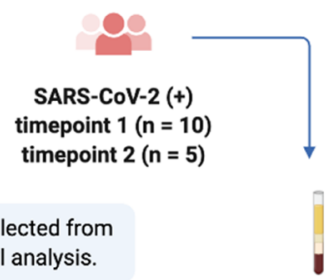
all subjects and stored at $-80^{\circ} \mathrm{C}$ until analysis.

Metabolite quantification by UHPLC-MS

Blood serum

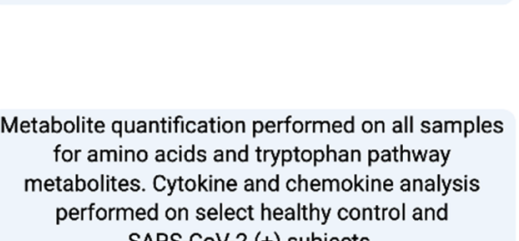

.
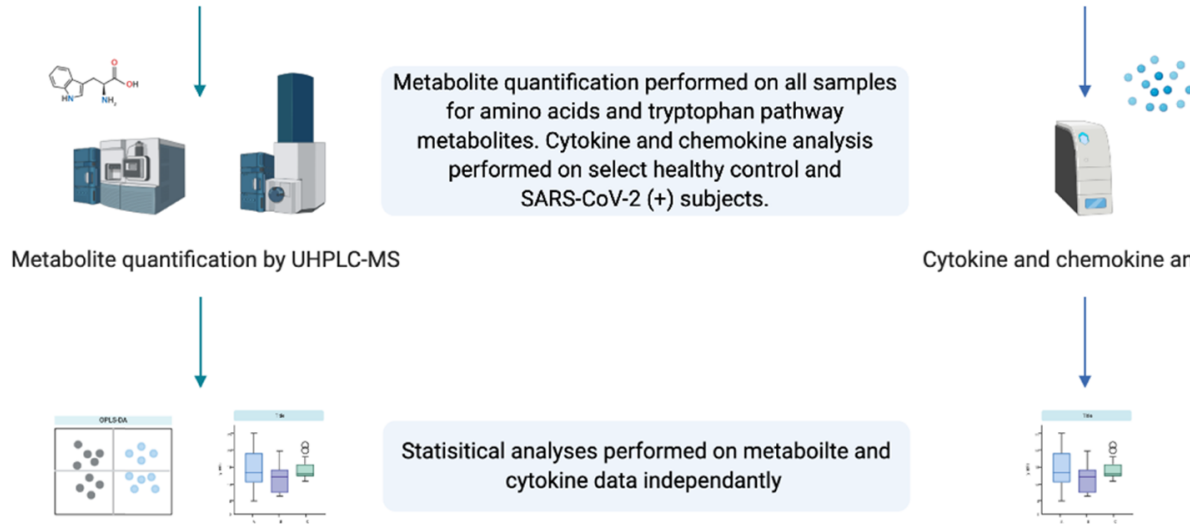

Statisitical analyses performed on metaboilte and cytokine data independantly Multivariate and univariate statistical analysis
(Healthy Controls $=16$, SARS-CoV- $2(-)=23$, SARS-CoV-2 $(+)=10)$
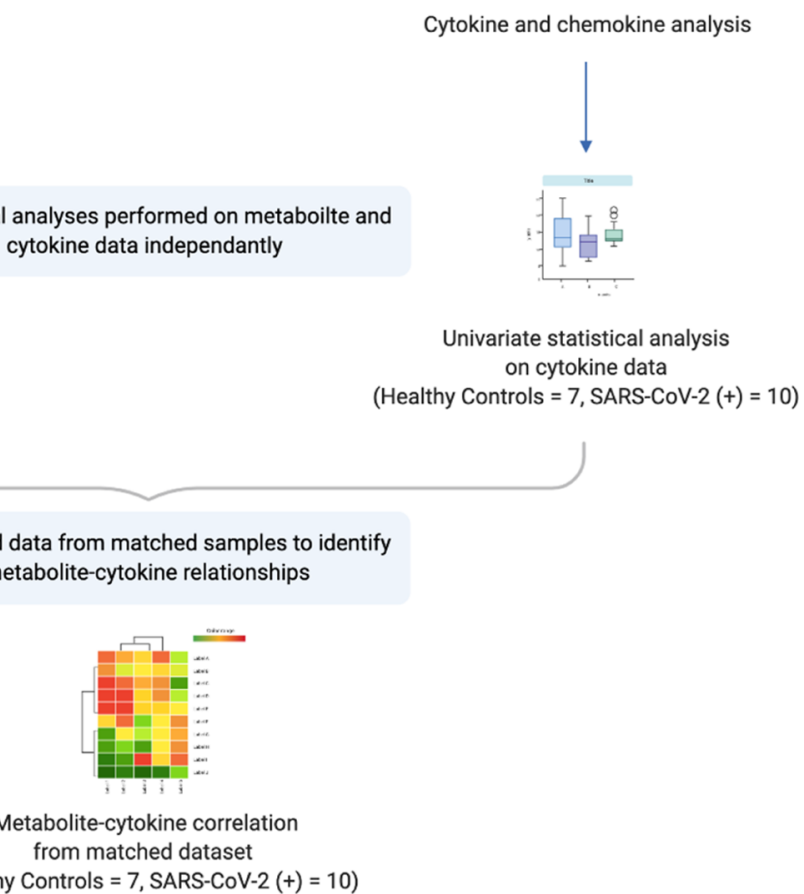

Combined data from matched samples to identify metabolite-cytokine relationships

(Healthy Controls $=7$, SARS-CoV-2 $(+)=10$ )

Figure 1. Overview of the study workflow including metabolite and cytokine analysis of blood plasma and serum collected from SARS-CoV-2 $(-)$, healthy control, and SARS-CoV-2 (+) individuals. Image was created using BioRender.com.

formate. After mixing, the samples were transferred to a Phenomenex PHREE phospholipid removal solid-phase extraction plate (Phenomenex, NSW, Australia). PHREE plates were then washed with an additional $150 \mu \mathrm{L}$ of methanol containing $2 \mathrm{mM}$ ammonium formate. Eluent collection plates were dried using a SpeedVac vacuum concentrator (Thermo Fisher, MA). Dried extracts were resuspended in $100 \mu \mathrm{L}$ of water with $0.1 \%$ formic acid prior to LC-MS analysis.

LC-MS analysis was performed using a Waters Acquity UPLC (Waters Corp., Milford, MA) coupled to a Waters Xevo TQ-XS MS (Waters Corp., Wilmslow, U.K.). Full instrument settings are described in the Supporting Information. Peak integration and calculation of resultant quantification data were performed using the TargetLynx package in MassLynx v4.2 (Waters Corp., Milford, MA). Details of additional methods can be found in the Supporting Information.

2.2.3. Cytokine and Chemokine Analysis. Where sample volume was sufficient, xMAP cytokine assays were carried out in 96-well polystyrene microplates using a
Luminex MagPix detection system (Luminex). A total of 34 cytokines and chemokines were quantified using a multiplexed Human Cytokines and Chemokines 34-Plex ProcartaPlex Panel 1A (Life Technologies) according to manufacturer's instructions. Briefly, capture bead mixes were incubated overnight with $25 \mu \mathrm{L}$ of serum sample or metabolite standards at $4{ }^{\circ} \mathrm{C}$ and then washed three times in a wash buffer; all washes were performed using a Bio-Plex Pro Wash station (Bio-Rad Laboratories). Detection antibody mixes were then added and incubated for $30 \mathrm{~min}$ at room temperature. The wells were subsequently washed three times before a reading buffer was added and incubated for a further $5 \mathrm{~min}$ at room temperature before analysis. Incubation steps were carried out using an orbital shaker at $500 \mathrm{rpm}$. Data were acquired and analyzed using the Luminex xPonent software.

\subsection{Statistical Analysis}

Supervised multivariate statistical modeling was performed with the combined set of 42 analytes (amino acids and tryptophan catabolic metabolites) using orthogonal projections to latent structure-discriminant analysis (OPLS-DA). Data were log-transformed and autoscaled prior to modeling. 


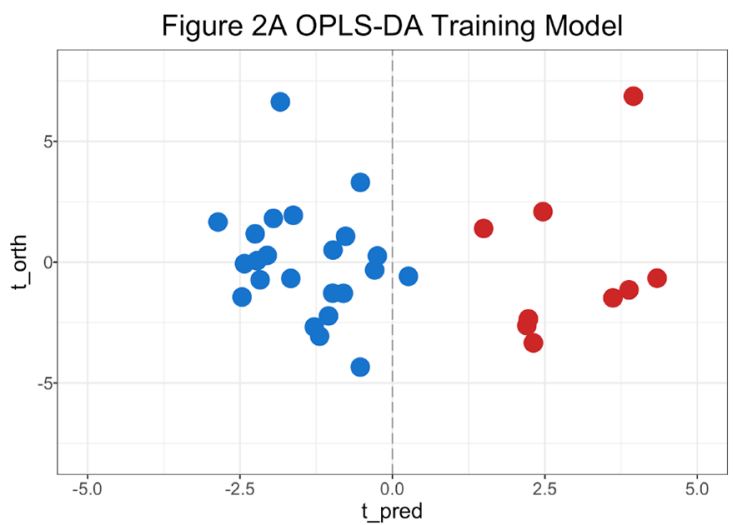

Figure 2B Projections

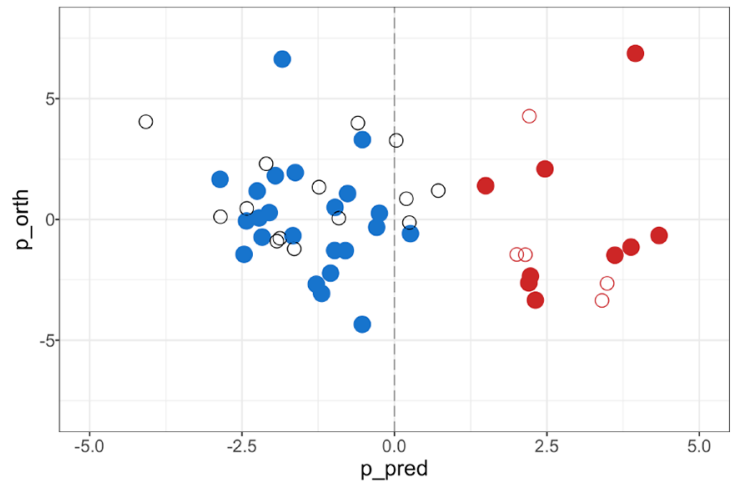

Figure 2C Projected SARS-CoV-2 (+) Case Study

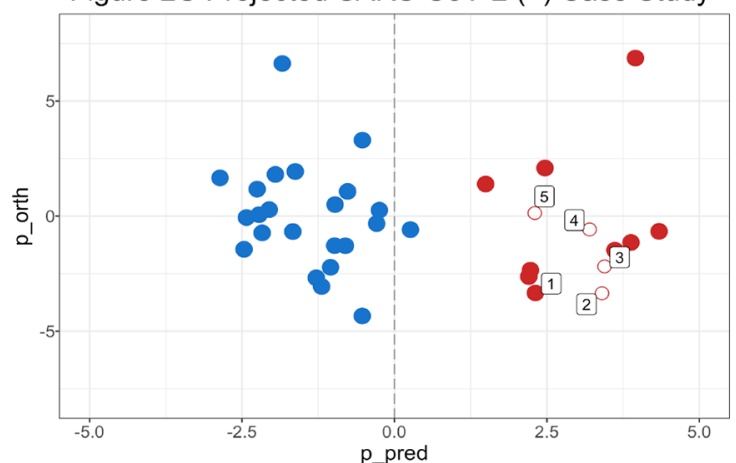

Figure 2D Projections SARS-CoV-2 (-) Hospitalized

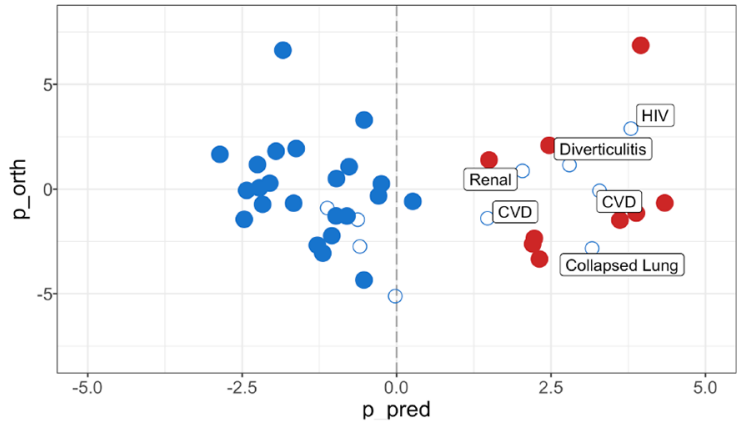

Figure 2E OPLS-DA Loadings

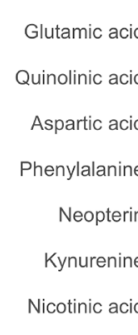

3-Hydroxykynurenine

Alanine-

Proline-

Taurine-

Lysine-

Ethanolamine

alpha-Aminobutyric acid

Glutamine-

Isoleucine-

1-Methylhistidine

Nicotinamide riboside

Glycine

Ornithine

Leucine-

Serine-

Tyrosine-

4-Hydroxyproline-

Kynurenic acid

Arginine-

Methionine-

Valine

3-Methylhistidine

Aminoadipic acid

Nicotinamide adenine dinucleotide-

5-Hydroxyindole acetic acid-

Threonine-

Asparagine-

Picolinic acid

Indole-3-acetic acid

Serotonin -

3-Hydroxyanthranilic acid-

$$
\begin{array}{r}
\text { Citrulline- } \\
\text { Xanthurenic acid- } \\
\text { Tryptophan } \\
\text { Histidine- }
\end{array}
$$

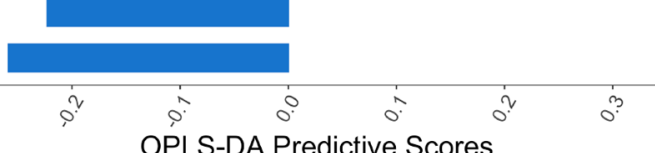

Training Set Projections

OPLS-DA Predictive Scores
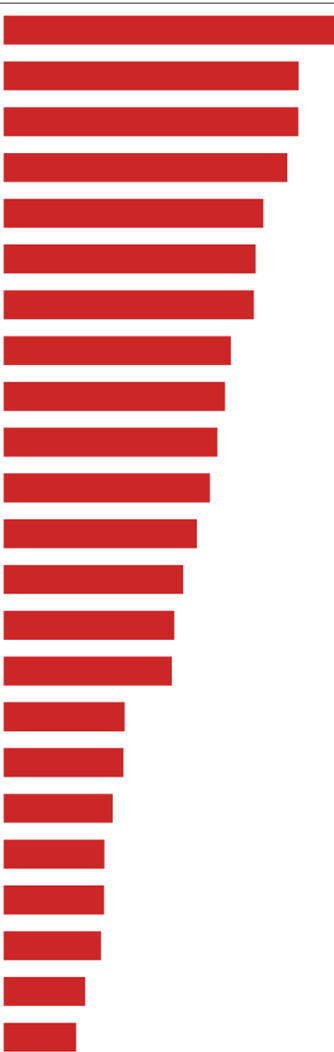

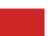
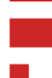
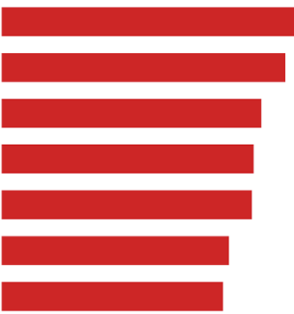

SARS-CoV-2 $(-)$ SARS-CoV- $2(-)$

- Sars-Cov-2(+) O SARS-CoV-2(+)

O Healthy Controls

Figure 2. OPLS-DA model visualizations. Scores plot showing training sample set of SARS-CoV-2 $(+) \mathrm{n}=10$ and SARS-CoV-2 (-) $\mathrm{n}=23(\mathrm{~A})$; note: only nine samples are visible due to two patients having similar prediction scores. Where available, a second timepoint of SARS-CoV-2 $(+)$ was projected for model validation $(\mathrm{n}=5)$ in addition to healthy controls $(\mathrm{n}=16)(\mathrm{B})$; a longitudinal hospitalized case study where five timepoints were collected and projected into the model (C); projection of 10 individuals who were hospitalized and underwent a routine SARS-CoV2 PCR testing and were diagnosed as SARS-CoV-2 (-) (D); predictive component loadings are shown in subplot (E). 

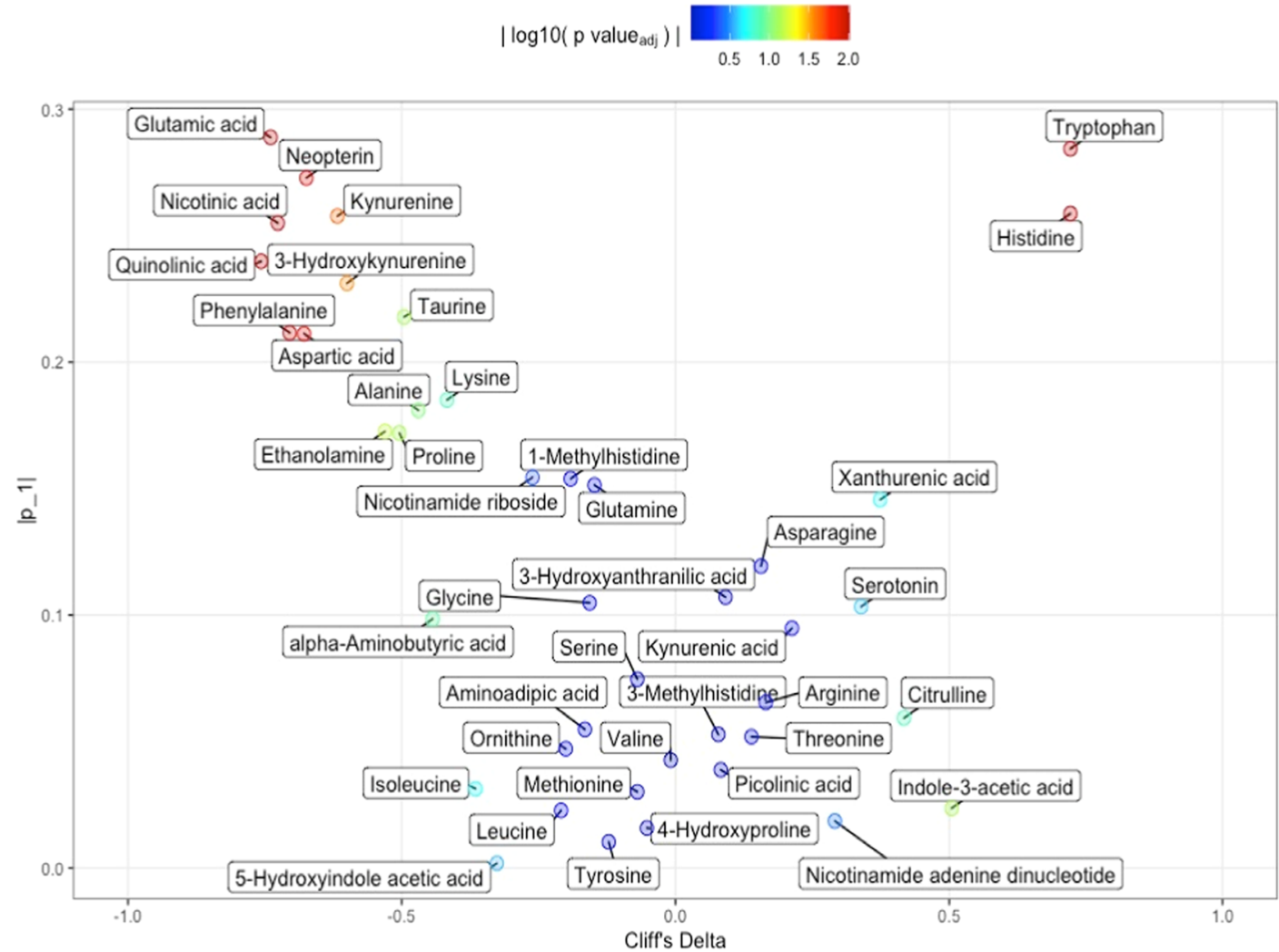

Figure 3. Eruption plot of the combined biogenic amines and tryptophan datasets for the SARS-CoV-2 (-) and SARS-CoV-2 (+) samples, formed from the Cliff's delta (abscissa) and the OPLS-DA loadings (ordinate). Variables are color-coded for statistical significance.

All computations were performed with statistical programming language R. Multivariate analysis was performed using the MetaboM8 package. ${ }^{31}$ The Cliff's delta statistic was applied to the 42 measured metabolites to assess group differences (i.e., SARS-CoV-2 (-) vs SARS-CoV-2 (+)). Absolute Cliff's delta scores were interpreted as 1 , indicating maximum difference and zero indicating no difference.

Mann-Whitney $U$ tests were performed on each of the 42 measured metabolites between two groups, SARS-CoV-2 $(-) / \mathrm{HC}$ and SARS-CoV-2 (+). To control the false discovery rate (FDR), $q$-values ${ }^{32}$ were generated from the MannWhitney $p$-values using the method proposed by Benjamini and Hochberg. ${ }^{33}$ The Mann-Whitney effect size estimates $(r)$ were applied to account for the bias from the small sample size. Estimates were interpreted as $>0.50,0.30$ to $<0.50$, and 0.10 to $<0.30$ corresponding to large, moderate, and small. Statistical significance was accepted at the level of $\alpha=0.05$.

For the panel of 14 cytokines, pairwise univariate analysis was completed using a two-sided Mann-Whitney $U$ test to compare between the $\mathrm{HC}$ and SARS-CoV-2 (+) groups (Table S1). Again, to control the false discovery rate (FDR), $q$ values were generated using the Benjamini and Hochberg method $^{33}$ and effect size estimates were generated using the Mann-Whitney $(r)$ test.

A hierarchical agglomerative cluster analysis was performed using Euclidean distances and Ward's minimal variance method as an objective function. Results were displayed using a circular dendrogram, with black and gray text colors indicating $q$-values below and above the significance threshold of 0.05 , respectively. A Spearman's correlation analysis was performed with cytokines and metabolite variables; the correlation coefficients are displayed in a heatmap, with rows and columns ordered according to clustering results. Prior to clustering, cytokine variables were log-transformed to approach normal distribution.

All statistics and data visualizations were generated in $\mathrm{R}$ (v.4.0.3) and R Studio (v.1.3.959).

\section{RESULTS}

To elucidate the underlying biochemistry of the host response to this complex mosaic disease, we focused our analysis on the relationship between SARS-CoV-2 infection, distinct metabolic pathways (i.e., amino acids and tryptophan catabolism) using a targeted panel of 42 metabolites, and the host inflammatory response through the measurement of 14 cytokines and chemokines (Figure 1).

To identify the metabolic signature associated with acute SARS-CoV-2 infection, supervised orthogonal projection to latent structure-discriminant analysis (OPLS-DA) was performed on metabolite data generated from SARS-CoV-2 (-) $(n=23)$ and SARS-CoV-2 $(+)(n=10)$ groups (Figure 2A). The OPLS-DA classified the two groups with AUROC $=0.94$ $\left(R^{2} X=0.15, R^{2} Y=0.78, Q^{2}=0.42\right)$. Model loadings with metabolites of importance are presented in Figure 2E. The Cliff's delta eruption plot (Figure 3) shows the combined biogenic amines and tryptophan metabolites and the comparative effect size differences between the SARS-CoV-2 $(-)$ and SARS-CoV-2 (+) groups. The plot emphasizes increased levels of neopterin, quinolinic acid, glutamic acid, kynurenine, and taurine in SARS-CoV-2 (+), whereas lower levels of tryptophan and histidine appear to drive the differences between the SARS-CoV-2 (-) and SARS-CoV-2 (+) groups. 
Table 1. Univariate Analysis of the SARS-CoV-2 Phenotyping Panel for 42 Quantified Amino Acid and Biogenic Amines ${ }^{a}$

\begin{tabular}{|c|c|c|c|c|c|c|c|c|}
\hline metabolite & $\begin{array}{l}\text { SARS-CoV-2 } \\
(-) \text { median } \\
\text { concentration } \\
(\mu \mathrm{M})\end{array}$ & $\begin{array}{l}\text { SARS-CoV-2 } \\
(+) \text { median } \\
\text { concentration } \\
(\mu \mathrm{M})\end{array}$ & $\begin{array}{c}\text { median } \\
\text { concentration } \\
\text { difference } \\
(\mu \mathrm{M})\end{array}$ & $\begin{array}{l}\text { lower } 95 \% \\
\text { confidence } \\
\text { interval }\end{array}$ & $\begin{array}{l}\text { upper } 95 \% \\
\text { confidence } \\
\text { interval }\end{array}$ & $\begin{array}{l}\text { Mann- } \\
\text { Whitney } p \\
\text { value }\end{array}$ & $\mathrm{BH} q$ value & $\begin{array}{c}\text { Mann- } \\
\text { Whitney } \\
\text { effect size }(r)\end{array}$ \\
\hline quinolinic acid & $1.10 \times 10^{0}$ & $2.50 \times 10^{0}$ & $1.30 \times 10^{0}$ & $-3.50 \times 10^{0}$ & $-7.10 \times 10^{-1}$ & $2.80 \times 10^{-5}$ & $7.20 \times 10^{-4}$ & $5.50 \times 10^{-1}$ \\
\hline glutamic acid & $5.60 \times 10^{1}$ & $1.30 \times 10^{2}$ & $7.80 \times 10^{1}$ & $-1.50 \times 10^{2}$ & $-4.40 \times 10^{1}$ & $4.40 \times 10^{-5}$ & $7.20 \times 10^{-4}$ & $5.40 \times 10^{-1}$ \\
\hline histidine & $8.40 \times 10^{1}$ & $5.40 \times 10^{1}$ & $-3.00 \times 10^{1}$ & $1.60 \times 10^{1}$ & $3.70 \times 10^{1}$ & $5.20 \times 10^{-5}$ & $7.20 \times 10^{-4}$ & $5.40 \times 10^{-1}$ \\
\hline tryptophan & $5.10 \times 10^{1}$ & $4.10 \times 10^{1}$ & $-9.30 \times 10^{0}$ & $5.40 \times 10^{0}$ & $1.90 \times 10^{1}$ & $2.00 \times 10^{-4}$ & $2.10 \times 10^{-3}$ & $5.00 \times 10^{-1}$ \\
\hline nicotinic acid & $4.10 \times 10^{-3}$ & $2.50 \times 10^{-2}$ & $2.10 \times 10^{-2}$ & $-4.30 \times 10^{-2}$ & $-6.50 \times 10^{-3}$ & $6.50 \times 10^{-4}$ & $4.60 \times 10^{-3}$ & $4.90 \times 10^{-1}$ \\
\hline aspartic acid & $4.00 \times 10^{0}$ & $8.30 \times 10^{0}$ & $4.30 \times 10^{0}$ & $-6.30 \times 10^{0}$ & $-1.30 \times 10^{0}$ & $4.20 \times 10^{-4}$ & $3.50 \times 10^{-3}$ & $4.80 \times 10^{-1}$ \\
\hline neopterin & $1.40 \times 10^{-2}$ & $3.70 \times 10^{-2}$ & $2.30 \times 10^{-2}$ & $-4.10 \times 10-2$ & $-6.70 \times 10^{-3}$ & $1.00 \times 10^{-3}$ & $6.00 \times 10^{-3}$ & $4.70 \times 10^{-1}$ \\
\hline kynurenine & $2.20 \times 10^{0}$ & $3.10 \times 10^{0}$ & $9.00 \times 10^{-1}$ & $-2.50 \times 10^{0}$ & $-4.50 \times 10^{-1}$ & $1.90 \times 10^{-3}$ & $9.00 \times 10^{-3}$ & $4.40 \times 10^{-1}$ \\
\hline phenylalanine & $6.40 \times 10^{1}$ & $8.20 \times 10^{1}$ & $1.80 \times 10^{1}$ & $-4.10 \times 10^{1}$ & $-6.00 \times 10^{0}$ & $1.90 \times 10^{-3}$ & $9.00 \times 10^{-3}$ & $4.30 \times 10^{-1}$ \\
\hline 3-hydroxykynurenine & $4.70 \times 10^{-2}$ & $7.60 \times 10^{-2}$ & $2.90 \times 10^{-2}$ & $-5.30 \times 10^{-2}$ & $-8.00 \times 10^{-3}$ & $3.30 \times 10^{-3}$ & $1.40 \times 10^{-2}$ & $4.20 \times 10^{-1}$ \\
\hline indole-3-acetic acid & $1.90 \times 10^{0}$ & $1.20 \times 10^{0}$ & $-6.90 \times 10^{-1}$ & $2.70 \times 10^{-1}$ & $1.20 \times 10^{0}$ & $3.60 \times 10^{-3}$ & $1.40 \times 10^{-2}$ & $4.20 \times 10^{-1}$ \\
\hline taurine & $6.50 \times 10^{1}$ & $1.00 \times 10^{2}$ & $4.00 \times 10^{1}$ & $-6.60 \times 10^{1}$ & $-8.60 \times 10^{0}$ & $7.40 \times 10^{-3}$ & $2.60 \times 10^{-2}$ & $3.80 \times 10^{-1}$ \\
\hline citrulline & $3.60 \times 10^{1}$ & $2.50 \times 10^{1}$ & $-1.10 \times 10^{1}$ & $2.40 \times 10^{0}$ & $1.80 \times 10^{1}$ & $1.10 \times 10^{-2}$ & $3.60 \times 10^{-2}$ & $3.60 \times 10^{-1}$ \\
\hline serotonin & $1.70 \times 10^{-1}$ & $8.90 \times 10^{-2}$ & $-7.60 \times 10^{-2}$ & $-1.20 \times 10^{-3}$ & $1.50 \times 10^{-1}$ & $5.40 \times 10^{-2}$ & $1.60 \times 10^{-1}$ & $2.80 \times 10^{-1}$ \\
\hline alanine & $3.30 \times 10^{2}$ & $4.20 \times 10^{2}$ & $8.90 \times 10^{1}$ & $-1.80 \times 10^{2}$ & $4.00 \times 10^{0}$ & $7.20 \times 10^{-2}$ & $2.00 \times 10^{-1}$ & $2.60 \times 10^{-1}$ \\
\hline xanthurenic acid & $1.50 \times 10^{-2}$ & $8.00 \times 10^{-3}$ & $-7.10 \times 10^{-3}$ & $-9.50 \times 10^{-4}$ & $1.40 \times 10^{-2}$ & $7.60 \times 10^{-2}$ & $2.00 \times 10^{-1}$ & $2.60 \times 10^{-1}$ \\
\hline ethanolamine & $7.30 \times 10^{0}$ & $8.90 \times 10^{0}$ & $1.60 \times 10^{0}$ & $-2.20 \times 10^{0}$ & $3.80 \times 10^{-1}$ & $9.90 \times 10^{-2}$ & $2.40 \times 10^{-1}$ & $2.40 \times 10^{-1}$ \\
\hline arginine & $8.00 \times 10^{1}$ & $5.90 \times 10^{1}$ & $-2.10 \times 10^{1}$ & $-6.90 \times 10^{0}$ & $3.60 \times 10^{1}$ & $1.30 \times 10^{-1}$ & $3.00 \times 10^{-1}$ & $2.20 \times 10^{-1}$ \\
\hline$\alpha$-aminobutyric acid & $1.80 \times 10^{1}$ & $2.60 \times 10^{1}$ & $7.40 \times 10^{0}$ & $-1.10 \times 10^{1}$ & $1.90 \times 10^{0}$ & $1.40 \times 10^{-1}$ & $3.00 \times 10^{-1}$ & $2.20 \times 10^{-1}$ \\
\hline lysine & $1.80 \times 10^{2}$ & $2.20 \times 10^{2}$ & $4.00 \times 10^{1}$ & $-1.00 \times 10^{2}$ & $1.80 \times 10^{1}$ & $1.80 \times 10^{-1}$ & $3.80 \times 10^{-1}$ & $1.90 \times 10^{-1}$ \\
\hline proline & $1.80 \times 10^{2}$ & $2.20 \times 10^{2}$ & $4.20 \times 10^{1}$ & $-8.50 \times 10^{1}$ & $1.60 \times 10^{1}$ & $2.00 \times 10^{-1}$ & $4.10 \times 10^{-1}$ & $1.80 \times 10^{-1}$ \\
\hline threonine & $1.30 \times 10^{2}$ & $1.20 \times 10^{2}$ & $-1.40 \times 10^{1}$ & $-1.40 \times 10^{1}$ & $4.40 \times 10^{1}$ & $2.10 \times 10^{-1}$ & $4.10 \times 10^{-1}$ & $1.80 \times 10^{-1}$ \\
\hline $\begin{array}{l}\text { 3-hydroxyanthranilic } \\
\text { acid }\end{array}$ & $7.10 \times 10^{-2}$ & $6.80 \times 10^{-2}$ & $-3.30 \times 10^{-3}$ & $-7.80 \times 10^{-3}$ & $3.70 \times 10^{-2}$ & $2.30 \times 10^{-1}$ & $4.20 \times 10^{-1}$ & $1.70 \times 10^{-1}$ \\
\hline aminoadipic acid & $1.80 \times 10^{0}$ & $2.40 \times 10^{0}$ & $5.20 \times 10^{-1}$ & $-1.30 \times 10^{0}$ & $3.90 \times 10^{-1}$ & $2.80 \times 10^{-1}$ & $4.80 \times 10^{-1}$ & $1.60 \times 10^{-1}$ \\
\hline asparagine & $5.30 \times 10^{1}$ & $5.10 \times 10^{1}$ & $-1.40 \times 10^{0}$ & $-3.00 \times 10^{0}$ & $1.30 \times 10^{1}$ & $2.80 \times 10^{-1}$ & $4.80 \times 10^{-1}$ & $1.60 \times 10^{-1}$ \\
\hline isoleucine & $6.40 \times 10^{1}$ & $6.80 \times 10^{1}$ & $4.10 \times 10^{0}$ & $-1.80 \times 10^{1}$ & $9.40 \times 10^{0}$ & $3.10 \times 10^{-1}$ & $4.80 \times 10^{-1}$ & $1.50 \times 10^{-1}$ \\
\hline $\begin{array}{l}\text { nicotinamide } \\
\text { riboside }\end{array}$ & $6.60 \times 10^{-1}$ & $7.20 \times 10^{-1}$ & $6.10 \times 10^{-2}$ & $-4.90 \times 10^{-1}$ & $1.50 \times 10^{-1}$ & $3.10 \times 10^{-1}$ & $4.80 \times 10^{-1}$ & $1.50 \times 10^{-1}$ \\
\hline 1-methylhistidine & $5.20 \times 10^{0}$ & $5.40 \times 10^{0}$ & $2.30 \times 10^{-1}$ & $-2.80 \times 10^{0}$ & $6.90 \times 10^{-1}$ & $3.40 \times 10^{-1}$ & $5.20 \times 10^{-1}$ & $1.40 \times 10^{-1}$ \\
\hline kynurenic acid & $4.00 \times 10^{-2}$ & $2.90 \times 10^{-2}$ & $-1.10 \times 10^{-2}$ & $-1.80 \times 10^{-2}$ & $1.80 \times 10^{-2}$ & $3.70 \times 10^{-1}$ & $5.40 \times 10^{-1}$ & $1.30 \times 10^{-1}$ \\
\hline ornithine & $6.90 \times 10^{1}$ & $9.20 \times 10^{1}$ & $2.30 \times 10^{1}$ & $-4.30 \times 10^{1}$ & $1.70 \times 10^{1}$ & $4.00 \times 10^{-1}$ & $5.40 \times 10^{-1}$ & $1.20 \times 10^{-1}$ \\
\hline picolinic acid & $3.60 \times 10^{-2}$ & $3.00 \times 10^{-2}$ & $-5.70 \times 10^{-3}$ & $-8.20 \times 10^{-3}$ & $1.90 \times 10^{-2}$ & $4.00 \times 10^{-1}$ & $5.40 \times 10^{-1}$ & $1.20 \times 10^{-1}$ \\
\hline glutamine & $6.70 \times 10^{2}$ & $6.50 \times 10^{2}$ & $-1.90 \times 10^{1}$ & $-9.10 \times 10^{1}$ & $1.70 \times 10^{2}$ & $4.70 \times 10^{-1}$ & $6.20 \times 10^{-1}$ & $1.10 \times 10^{-1}$ \\
\hline 3-methylhistidine & $6.80 \times 10^{0}$ & $4.60 \times 10^{0}$ & $-2.20 \times 10^{0}$ & $-3.10 \times 10^{0}$ & $5.50 \times 10^{0}$ & $5.00 \times 10^{-1}$ & $6.40 \times 10^{-1}$ & $9.90 \times 10^{-2}$ \\
\hline $\begin{array}{l}\text { 5-hydroxyindole } \\
\text { acetic acid }\end{array}$ & $5.50 \times 10^{-2}$ & $5.90 \times 10^{-2}$ & $3.90 \times 10^{-3}$ & $-2.30 \times 10^{-2}$ & $1.60 \times 10^{-2}$ & $6.50 \times 10^{-1}$ & $7.80 \times 10^{-1}$ & $6.70 \times 10^{-2}$ \\
\hline leucine & $1.20 \times 10^{2}$ & $1.20 \times 10^{2}$ & $1.70 \times 10^{0}$ & $-2.80 \times 10^{1}$ & $2.40 \times 10^{1}$ & $6.50 \times 10^{-1}$ & $7.80 \times 10^{-1}$ & $6.70 \times 10^{-2}$ \\
\hline glycine & $2.20 \times 10^{2}$ & $2.20 \times 10^{2}$ & $8.70 \times 10^{-1}$ & $-5.40 \times 10^{1}$ & $3.30 \times 10^{1}$ & $7.40 \times 10^{-1}$ & $8.60 \times 10^{-1}$ & $5.00 \times 10^{-2}$ \\
\hline valine & $2.30 \times 10^{2}$ & $2.20 \times 10^{2}$ & $-8.90 \times 10^{0}$ & $-2.90 \times 10^{1}$ & $5.00 \times 10^{1}$ & $7.60 \times 10^{-1}$ & $8.60 \times 10^{-1}$ & $4.60 \times 10^{-2}$ \\
\hline serine & $1.00 \times 10^{2}$ & $9.90 \times 10^{1}$ & $-9.40 \times 10^{-1}$ & $-1.80 \times 10^{1}$ & $2.40 \times 10^{1}$ & $7.80 \times 10^{-1}$ & $8.60 \times 10^{-1}$ & $4.30 \times 10^{-2}$ \\
\hline tyrosine & $6.70 \times 10^{1}$ & $7.30 \times 10^{1}$ & $5.70 \times 10^{0}$ & $-1.60 \times 10^{1}$ & $1.40 \times 10^{1}$ & $8.20 \times 10^{-1}$ & $8.80 \times 10^{-1}$ & $3.50 \times 10^{-2}$ \\
\hline methionine & $2.40 \times 10^{1}$ & $2.60 \times 10^{1}$ & $1.70 \times 10^{0}$ & $-4.60 \times 10^{0}$ & $5.70 \times 10^{0}$ & $8.70 \times 10^{-1}$ & $9.20 \times 10^{-1}$ & $2.50 \times 10^{-2}$ \\
\hline $\begin{array}{l}\text { nicotinamide } \\
\text { adenine } \\
\text { dinucleotide }\end{array}$ & $4.80 \times 10^{-3}$ & $5.00 \times 10^{-3}$ & $1.50 \times 10^{-4}$ & $-2.60 \times 10^{-3}$ & $2.40 \times 10^{-3}$ & $9.20 \times 10^{-1}$ & $9.30 \times 10^{-1}$ & $1.60 \times 10^{-2}$ \\
\hline 4-hydroxyproline & $1.10 \times 10^{1}$ & $1.10 \times 10^{1}$ & $1.80 \times 10^{-1}$ & $-4.50 \times 10^{0}$ & $3.20 \times 10^{0}$ & $9.30 \times 10^{-1}$ & $9.30 \times 10^{-1}$ & $1.40 \times 10^{-2}$ \\
\hline
\end{tabular}

${ }^{a}$ Statistical comparisons were performed with the Mann-Whitney $U$ test. To control the false discovery rate (FDR), $q$ values were generated from the Mann-Whitney $U$ test $p$ values using the method proposed by Benjamini and Hochberg. Boxplots of the data are presented in Figures 4 and S1.

To test the predictivity of the model, data acquired from samples collected at a second timepoint from a subset of the SARS-CoV-2 $(+)$ group $(n=5)$ and samples collected from healthy controls $(n=16)$ were then projected onto the model, with $100 \%$ of the additional samples classified correctly (Figure 2B). Following the observed agreement between SARS-CoV-2 (-) and healthy controls, the two groups were combined for the subsequent univariate analysis. A SARS-
CoV-2 $(+)$ case study where multiple timepoints $(n=5)$ were collected over a period of 19 days from the same individual were projected to observe longitudinal disease trajectory until hospital discharge (Figure 2C).

The model was further examined by projecting a group of hospitalized participants who were either admitted for COVID-19-like symptoms, but subsequently tested negative for the virus and were discharged without treatment $(n=4)$, 

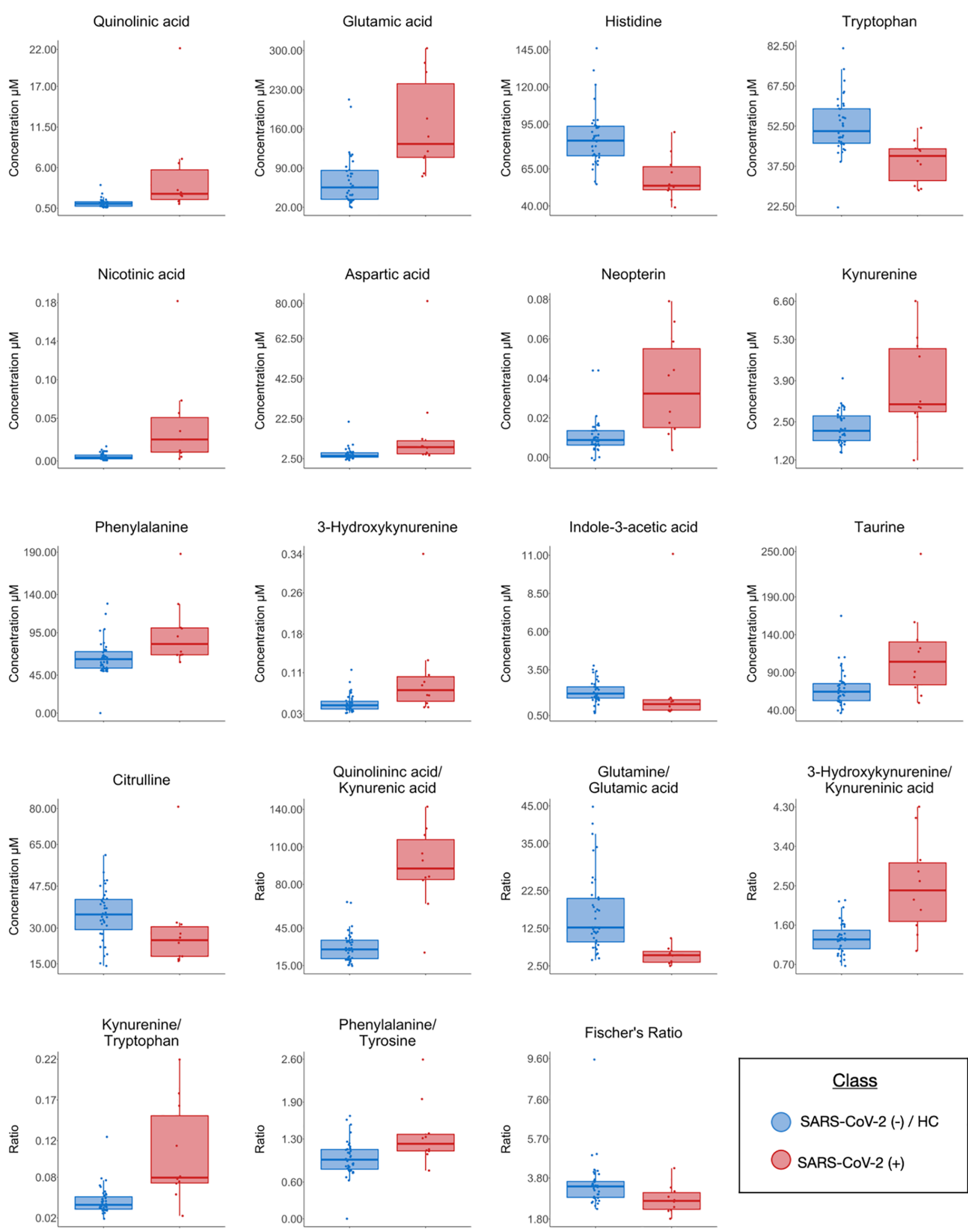

Figure 4. Two group boxplot comparisons of the plasma metabolites that were present at significantly different concentrations in the MannWhitney univariate testing $(p<0.05)$. The SARS-CoV-2 $(-)$ and HC groups were combined into a single group for univariate analysis based on their similarity in the OPLS-DA multivariate models (Figure 2). Fischer's ratio was calculated as the ratio of branched-chain amino acids to aromatic amino acids. The remaining nonsignificant metabolites are presented as two group box plots in Figure S1. Additional three group boxplots representing HC, SARS-CoV-2 (-), and SARS-CoV-2 (+) groups as separate entities are presented in Figures S2 and S3.

or were receiving treatment for a range of multiorgan diseases ( $n=6)$, including collapsed lung, diverticulitis, and cardiovascular disease and tested negative for SARS-CoV-2 infection in routine hospital screening. The individuals who were incorrectly admitted for COVID-19 clustered with the SARS-CoV-2 (-) group in the model. However, the 
(A)

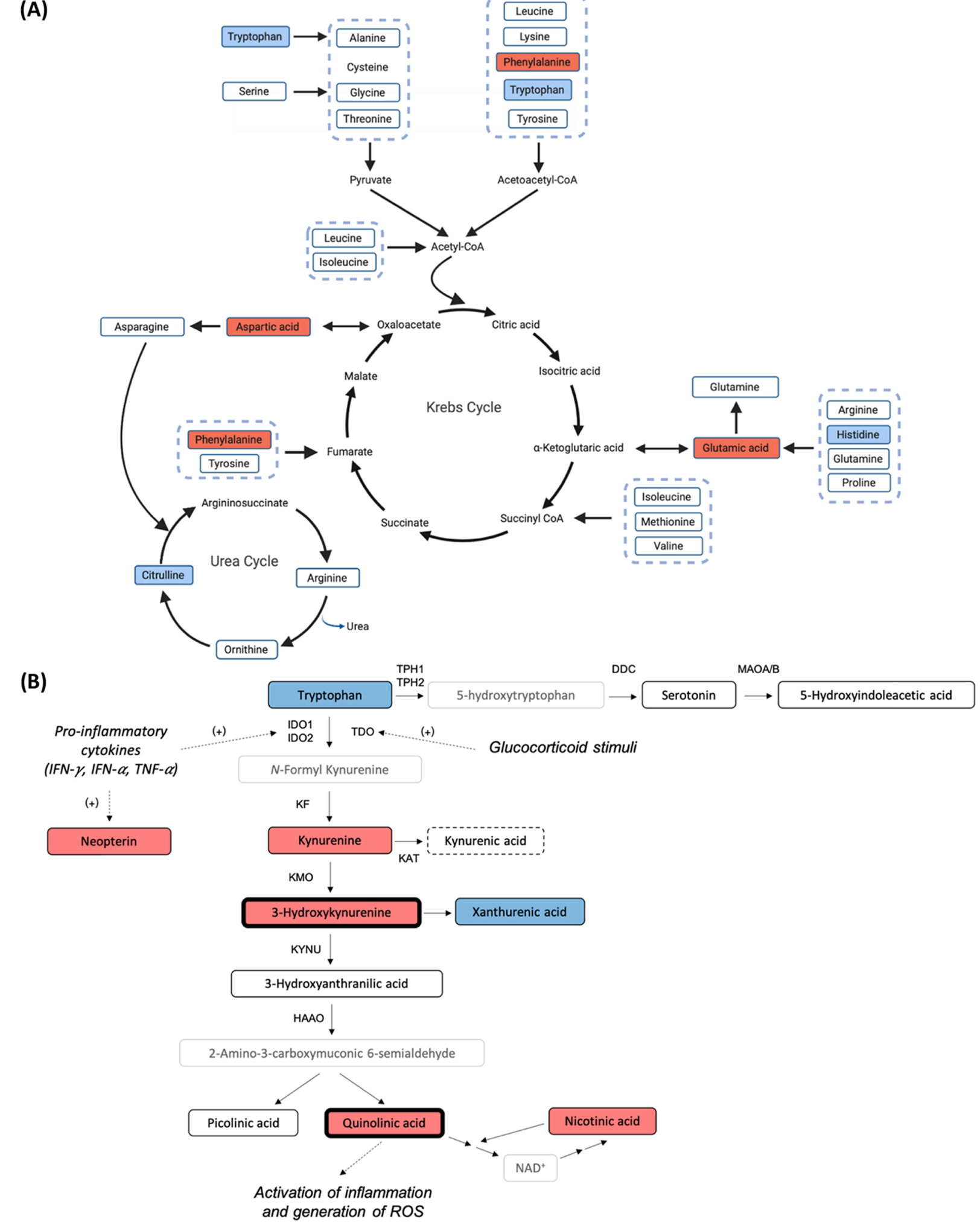

Figure 5. Metabolic pathway associations of significantly different metabolites in COVID-19. (A) Alterations in amino acid metabolism associated with SARS-CoV-2 (+) infection. Blue boxes indicate a significant decrease in SARS-CoV-2 (+) patients; red boxes indicate a significant increase $(p<0.05)$ in SARS-CoV-2 (+) patients. (B) Schematic presentation of the kynurenine pathway of tryptophan metabolism. Blue boxes indicate a significant decrease in SARS-CoV-2 $(+)$ patients; red boxes indicate a significant increase $(p<0.05)$ in SARS-CoV-2 $(+)$ patients; bold boxes indicate metabolites previously associated with neurotoxicity; dashed boxes indicate metabolites with neuroprotective properties; faded boxes indicate metabolites not measured in this analysis. Abbreviations: IFN- $\gamma$, interferon- $\gamma$; IFN- $\alpha$, interferon- $\alpha$; TNF- $\alpha$, tumor necrosis factor- $\alpha$; AHR, aryl hydrocarbon receptor; TPH1; tryptophan hydroxylase 1; TPH2, tryptophan hydroxylase 2; DDC, 3,4-dihydroxyphenylalanine (DOPA) decarboxylase; MAOA, monoamine oxidase A; MAOB monoamine oxidase B; IDO1, indoleamine-2,3-dioxygenase 1; IDO2, indoleamine-2,3dioxygenase 2; TDO2, tryptophan2,3-dioxygenase; KF, kynurenine formamidase; KAT, kynurenine aminotransferase; KMO, kynurenine 3monooxygenase; KYNU, kynurenine hydroxylase; HAAO, 3-hydroxyanthranilic acid dioxygenase. Image created with BioRender.com. 
hospitalized patients receiving treatment for other diseases were projected alongside the SARS-CoV-2 (+) group (Figure 2D).

From the panel of 42 metabolites investigated, 13 were univariately significant $(p<0.05$ Mann-Whitney $U$ test, with the false-discovery rate controlled by the BenjaminiHochberg method) between the SARS-CoV-2 (-)/HC and SARS-CoV-2 $(+)$ groups (Table 1$)$. Higher concentrations of quinolinic acid $\left(p=2.81 \times 10^{-5}\right)$, glutamic acid $(p=4.45 \times$ $\left.10^{-5}\right)$, nicotinic acid $\left(p=6.50 \times 10^{-4}\right)$, aspartic acid $(p=4.22$ $\left.\times 10^{-4}\right)$, neopterin $\left(p=1.00 \times 10^{-3}\right)$, kynurenine $(p=1.93 \times$ $\left.10^{-3}\right)$, phenylalanine $\left(p=1.90 \times 10^{-3}\right)$, 3-hydroxykynurenine $\left(p=3.28 \times 10^{-3}\right)$, and taurine $\left(p=7.38 \times 10^{-3}\right)$, while lower concentrations of histidine $\left(p=5.16 \times 10^{-5}\right)$, tryptophan $(p=$ $\left.2.03 \times 10^{-4}\right)$, indole-3-acetic acid $\left(p=3.56 \times 10^{-3}\right)$, and citrulline $\left(p=1.10 \times 10^{-2}\right)$ were observed in the SARS-CoV$2(+)$ group (Figure 4 and Table 1). Amino acid and tryptophan pathway schematics highlight the significantly altered metabolites in Figure 5A,B, respectively.

Furthermore, key metabolite ratios demonstrated significant changes between the SARS-CoV-2 (-)/HC and SARS-CoV-2 (+) groups (Table 2 and Figure 4 ). The quinolinic acid/ kynurenic acid ratio $\left(p=2.80 \times 10^{-6}\right)$, 3-hydroxykynurenine/ kynurenic acid ratio $\left(p=2.30 \times 10^{-4}\right)$, kynurenine/ tryptophan ratio $\left(p=2.61 \times 10^{-4}\right)$, and phenylalanine/ tyrosine ratio $\left(p=9.42 \times 10^{-3}\right)$ were found to be increased in the SARS-CoV-2 $(+)$ group, while the glutamine/glutamic acid ratio $\left(p=6.03 \times 10^{-6}\right)$ and branch chain amino acid/ aromatic amino acid ratio (Fischer's ratio, $p=1.02 \times 10^{-2}$ ) were decreased in the SARS-CoV-2 (+) group.

Hierarchical clustering of the 42 metabolites revealed seven individual clusters (clusters A-G, Figure 6), which were grouped based on metabolite similarity and association with SARS-CoV-2 infection. Of significance, cluster B comprised aspartic acid, glutamic acid, phenylalanine, and taurine; cluster C comprised 3-hydroxykynurenine, quinolinic acid, nicotinic acid, kynurenine, and neopterin; and cluster F comprised citrulline, histidine, tryptophan, and indole-3-acetic acid.

Following the identification of a metabolic signature of SARS-CoV-2 infection, the relationship with host inflammatory cytokine and chemokine response in COVID-19 was explored. Soluble immune mediators were investigated for differences between the $\mathrm{HC}$ and SARS-CoV-2 (+) groups. The SARS-CoV-2 (-) group did not undergo cytokine and chemokine analysis due to sample availability. Univariate Mann-Whitney $U$ tests revealed higher concentrations in the SARS-CoV-2 $(+)$ group of IL-1 RA $\left(p=1.03 \times 10^{-2}\right)$, IP-10 $\left(p=1.37 \times 10^{-2}\right), \operatorname{SDF}-1 \alpha\left(p=2.58 \times 10^{-2}\right), \mathrm{IL}-8(p=2.58$ $\left.\times 10^{-2}\right), \operatorname{TNF}-\alpha\left(p=3.04 \times 10^{-2}\right), \operatorname{MIP}-1 \beta(p=3.17 \times$ $\left.10^{-2}\right)$, and MCP-1 $\left(p=3.17 \times 10^{-2}\right)$ (Table S2 and Figure $\mathrm{S} 1)$. Correlation analysis revealed metabolic/soluble immune mediator relationships in the host response to SARS-CoV-2 infection and are described in detail in Figure 7. Of specific interest, metabolites from clusters $\mathrm{B}$ and $\mathrm{C}$ were positively associated with SDF- $1 \alpha$ and MIP- $1 \beta$ (neopterin and phenylalanine); IP-10, RANTES, and IL-18 (kynurenine and 3hydroxykynurenine); and IFN- $\gamma$ and MCP-1 (kynurenine and 3-hydroxykynurenine). Negative correlations in the dataset were observed between cluster F and SDF- $1 \alpha$, MIP-1 $\beta$, IP-10, and IL-1RA (citrulline and histidine).

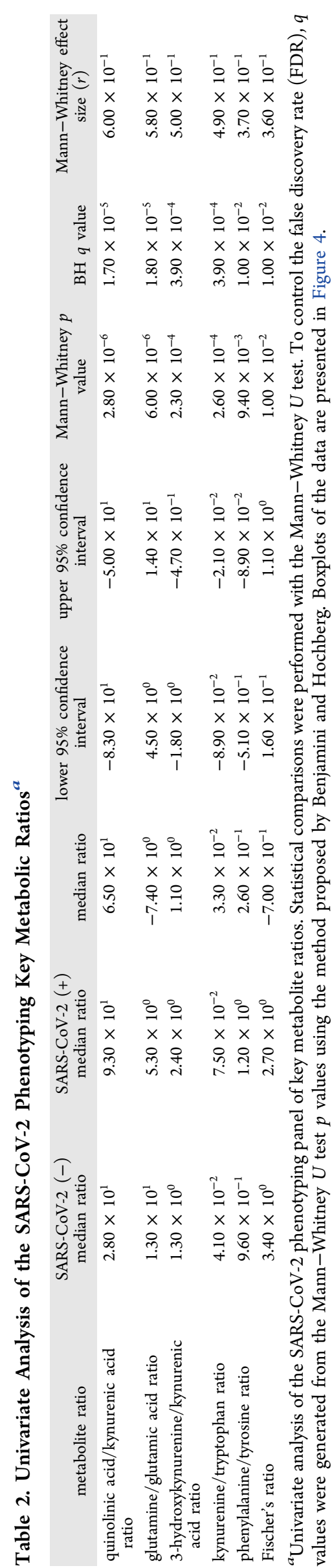




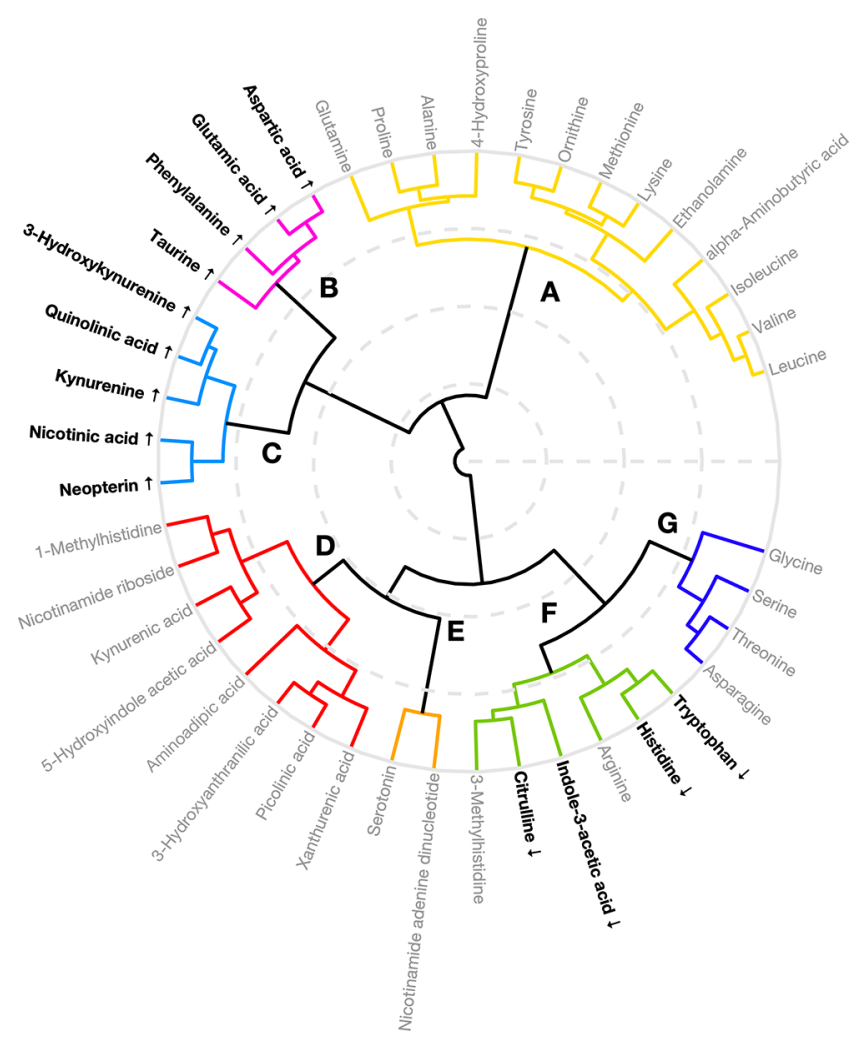

Figure 6. Hierarchical cluster analysis (HCA) visualized as a circular dendrogram grouping individual metabolites detected in amino acid and tryptophan assays. Label coloring is based on the univariate analysis between SARS-CoV-2 (-)/HC and SARS-CoV-2 (+); (Table $1, q$-value). Black text indicates $q$-value of $<0.05$; arrows indicate directionality of plasma concentration change in SARS-CoV$2(+)$ patients.

\section{DISCUSSION}

The current study sought to describe key metabolic pathways that underpin host responses to SARS-CoV-2 infection and identify critical cytokine and chemokine relationships that occur during infection. Four groups of participants were classified from our sample cohort.

Multivariate OPLS-DA modeling of the metabolite panel revealed that individuals infected with SARS-CoV-2 displayed a phenotypic signature compared to the SARS-CoV-2 (-) group (Figure 2A). The result indicated that a systemic phenoconversion occurs, which can be detected and quantified using the mass spectrometry approach described. This concept of a phenoconversion is supported by the subsequent projection of data acquired from samples of healthy volunteers alongside secondary timepoint samples collected from the SARS-CoV-2 (+) group. The healthy controls were classified with the SARS-CoV-2 (-) group in the model, while the second timepoints are all classified with the SARS-CoV-2 (+) group in the model (Figure 2B). Interestingly, multiple timepoints from an individual case study mapped onto the model showing an apparent metabolic journey through infection, from admission at timepoint 1 with initial symptoms, peaking in the metabolic space at timepoint 3 (day 12) and shifting back toward the healthy metabolic space on hospital discharge at timepoint 5 (day 19) (Figure 2C).

When projecting data onto the model collected from hospitalized individuals, two distinct groups were formed
(Figure 2D). The first was classified with the SARS-CoV-2 (-) samples in the model. These samples were subsequently revealed to be individuals admitted to hospital with presumed COVID-19 disease but tested negative for SARS-CoV-2 and were discharged. The remaining samples clustered with the SARS-CoV-2 $(+)$ group and were revealed to be individuals receiving hospital treatment for additional conditions, and had tested negative for SARS-CoV-2 in routine hospital screening. The similarity between the signature and the multiorgan nature of disease within the patients detailed in Figure 2D seems to indicate and support the fact that COVID-19 presents as a complex mosaic disease. Further studies are required to investigate infected individuals in tandem with those with multifactorial diseases to further understand if a specific unique phenotype of SARS-CoV-2 infection occurs or if it is a result of the overall systemic impact of the disease.

Following multivariate modeling, hierarchical cluster and correlation analyses (Figures 6 and 7) were completed on the metabolic and cytokine data acquired within the dataset. This analysis revealed key metabolite clusters that were perturbed during the course of infection, along with metabolite/cytokine associations that are part of the host response. The resultant clusters again indicated a systemic multiorgan impact of infection, supporting previously reported clinical observations in the disease. For example, recent studies have highlighted an increasing trend of neurological complications associated with SARS-CoV-2 infection. ${ }^{8-10}$ Interestingly, cluster C demonstrated increases in quinolinic acid and 3-hydroxykynurenine in SARS-CoV-2 (+) patients, both metabolites have been reported to exhibit neurotoxic properties in vitro. ${ }^{34-37}$ Furthermore, the ratio between both metabolites and the neuroprotective kynurenic acid was also found to increase in the SARS-CoV-2 (+) group, both of which have specifically been referred to as neurotoxic ratios in the literature. ${ }^{38,39}$ Quinolinic acid is thought to be a neuronal excitotoxin, acting as an agonist for glutamate receptors exciting neurons; exaggerated stimulation leads to neuronal damage through multimechanistic neurotoxicity, ${ }^{35,36,40}$ while 3-hydroxykynurenine is thought to exert its neurotoxicity through the formation of free radicals. ${ }^{40}$ This unfavorable balance of metabolites with potential neurotoxic properties has previously been reported following central nervous system (CNS) infections including encephalitis and viral meningitis ${ }^{34,41}$ as well as diseases of neurodegeneration, including Parkinson's disease, ${ }^{38}$ Alzheimer's' disease, ${ }^{42}$ and Huntington's disease. ${ }^{43}$ The most consistently reported persistent neurological symptoms with SARS-CoV-2 infection are anosmia and ageusia, also common features of Parkinson's disease, ${ }^{44}$ together with persistent fatigue following infection. ${ }^{45}$ Fatigue is the most common symptom in systemic lupus erythematosus (SLE), which has remarkable similarity in the kynurenine metabolism perturbations noted here. ${ }^{46}$ Of added interest, although not found to be significant, the concentration of serotonin was observed to be lower in SARSCoV-2 (+) individuals (Figure S1). Considering the implication of the tryptophan metabolic pathway in depression and the reported persistent symptoms of COVID-19 syndrome including depression, anxiety, and fatigue, this finding also may be associated with long-lasting neuropsychiatric consequences. Furthermore, both quinolinic acid and 3-hydroxykynurenine correlated with the chemokine MCP1/CCL2 (Figure 7), a mediator responsible for leukocyte attraction and neuroinflammation in the CNS. ${ }^{47}$ The presence 


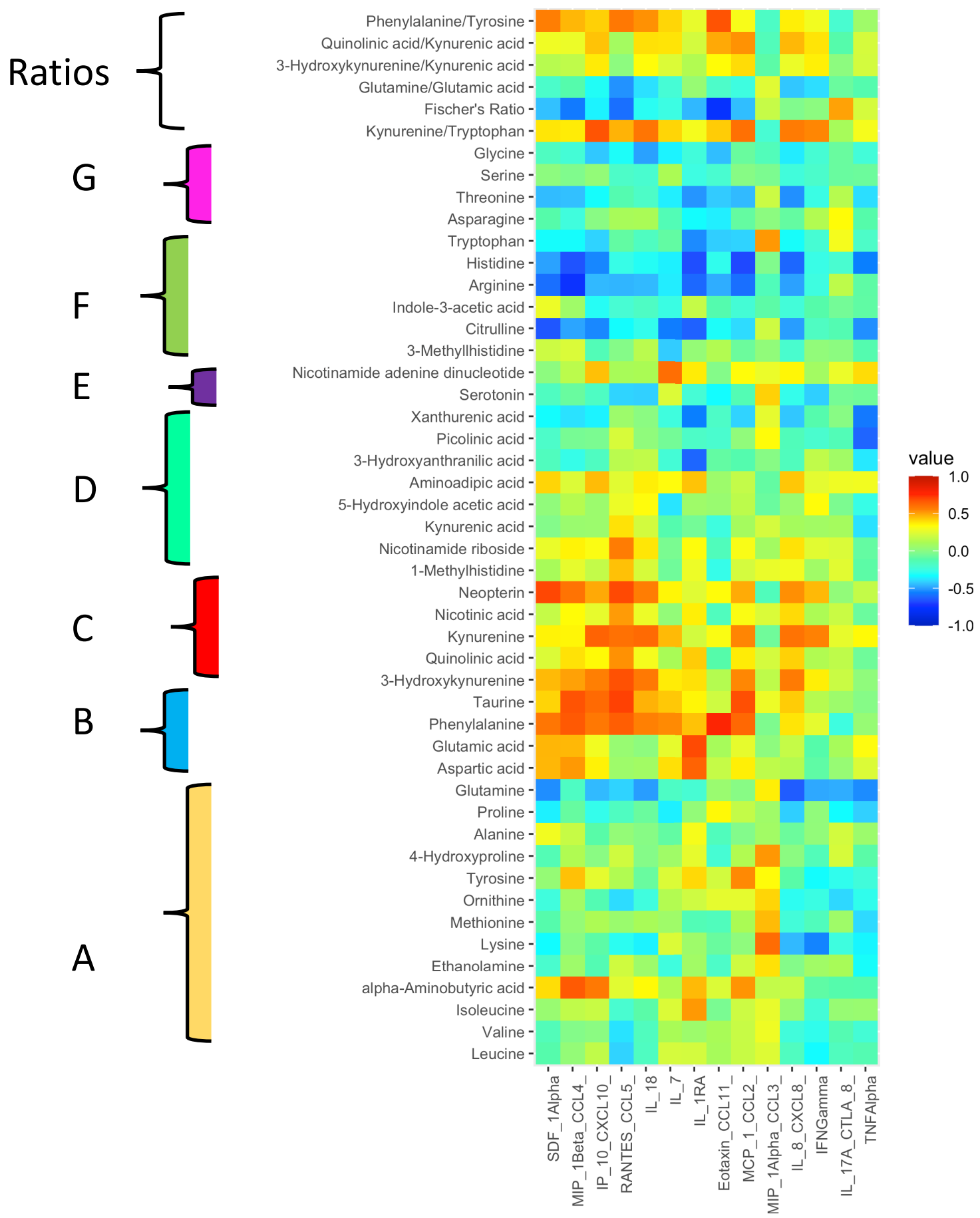

Figure 7. Correlation heatmap of cytokines and chemokines $(n=14)$ and plasma metabolites $(n=42)$. Spearman's correlation coefficients were calculated for each metabolite and soluble immune soluble mediators. Row represents clustering order from Figure 4, indicated by colored parenthesis. Column order is determined from Euclidean distances using Ward's clustering method.

of MIP- $1 \beta$, another chemoattractant immune regulator, demonstrated significantly higher concentrations in the SARS-CoV-2 (+) group (Table S2 and Figure S4), which indicates that MIP- $1 \beta$ may also participate in the neuroinflammatory response to viral infection. These chemokine correlations hint at a deeper systemic metabolic role in the host infection response for both quinolinic acid and 3hydroxykynurenine.

Cluster C also contains kynurenine and neopterin, both of which were found in these data at higher concentrations in the SARS-CoV-2 (+) group compared to the HC and SARS-CoV-
2 (-) groups. IDO is a key enzyme thought to regulate immune response in the peripheral system ${ }^{48}$ and act as a mechanistic indicator of the overall antimicrobial activity of interferons. $^{49,50}$ Furthermore, IDO has been shown to undergo upregulation following the release of IFN- $\gamma$ by monocytes, macrophages, and T-cells following viral infection. $^{51,52}$ IFN- $\gamma$ was also present at significantly higher concentrations in the SARS-CoV-2 $(+)$ group, and therefore, activation of IDO by the interferon may help explain the observations of higher concentrations of kynurenine and its neurotoxic catabolites discussed previously. Additional support 
of IDO activation is provided by the kynurenine/tryptophan ratio, which is significantly increased with SARS-CoV-2 infection. Furthermore, the IFN- $\gamma$ relationship with cluster $\mathrm{C}$ continues with neopterin, as the interferon induces the production and release of the metabolite in monocytes and macrophages. Neopterin, therefore, is thought to be a marker of the degree of cellular immune activation. ${ }^{53}$ In these data, both kynurenine and neopterin concentrations correlated with IFN- $\gamma$ (Figure 5), indicating an intertwined relationship in the inflammatory host response to SARS-CoV-2 infection. Furthermore, RANTES, IP-10, IL-18, and IL-8 are associated with inflammatory and cytotoxic $\mathrm{T}$ cells and were strongly associated with 3-hydroxykynurenine, kynurenine, and neopterin, indicating that these circulating cytokines reflect the severity of the respiratory infection.

Cementing its role as a key metabolic cluster in response to infection, cluster $\mathrm{C}$ also contained nicotinic acid. Nicotinic acid, increased in SARS-CoV-2 (+), is part of the kynurenine pathway (Figure 5B) and is thought to influence the immune response, with reported anti-inflammatory properties in monocytes and macrophages, which express nicotinic acid receptors. ${ }^{54}$ The amine derivative of nicotinic acid, nicotinamide, has recently been reported to be disrupted in the following SARS-CoV-2 infection, ${ }^{55}$ supporting the observation. Interestingly, nicotinic acid has been reported to be a mechanistic metabolite in response to dyslipidemia by raising high-density lipoprotein (HDL) and lowering low-density lipoprotein (LDL), very-low-density lipoprotein (VLDL), and triglycerides via activation of peroxisome proliferator-activated receptors (PPARs), ${ }^{56}$ which in turn play a role in the regulation of energy homeostasis. However, although dyslipidemia has been reported in SARS-CoV-2 infection, it was found to accompany low HDL and high LDL, ${ }^{21,57}$ therefore suggesting that the increase in nicotinic acid may be part of a wider host compensatory mechanism to normalize HDL and LDL concentrations.

Previous analysis of the WA SARS-CoV-2 (+) cohort, using an integrated multiplatform metabolic phenotyping approach, revealed dyslipidemia and lipoprotein abnormalities and also indicated a hepatic impact of viral infection. ${ }^{21}$ This appears to be supported by the metabolites found in cluster B (Figure 6), which contained phenylalanine, taurine, glutamic acid, and aspartic acid. Higher concentrations of phenylalanine, taurine, glutamic acid, and aspartic acid have been reported as indicators of liver disease, including hepatic fibrosis, ${ }^{58}$ acute hepatic failure, ${ }^{29,59}$ and hepatic encephalopathy. ${ }^{60,61}$ This is further supported in these data by observations of a lower Fischer's ratio in the SARS-CoV-2 (+) group. Fischer's ratio is the molar ratio of branched chain to aromatic amino acids, which is an important clinical calculation for assessing liver metabolism and hepatic function. ${ }^{61}$ Observations of hepatic abnormality are consistent with previous studies of SARSCoV-1, MERS-CoV, and SARS-CoV-2, indicating that liver injury may be occurring during the infection period with a high prevalence of abnormal aminotransferase enzymes. Systemic elevations in taurine are known to be associated with hepatotoxicity caused by a variety of drugs and model toxins $^{62}$ and in cardiac ischemia. ${ }^{63}$

The metabolites from cluster $\mathrm{B}$ are also associated with proinflammatory cytokines and chemokines including SDF- $1 \alpha$, MIP-1 $\beta$, IP-10, and RANTES (Table 2), all of which are involved in the promotion of inflammatory immune responses. This relationship is likely mediated by the metabolic influences of neopterin in instances of systemic inflammation. As previously discussed, proinflammatory stimuli lead to the upregulation of neopterin, which impacts the bioavailability of the enzymatic co-factor tetrahydrobiopterin $\left(\mathrm{BH}_{4}\right)$, subsequently disrupting the conversion of phenylalanine to tyrosine by phenylalanine 4-hydroxylase (PAH). ${ }^{64}$ This relationship is reflected in these data, with a significantly higher phenylalanine/tyrosine ratio present in the SARS-CoV-2 (+) group (Table 1 and Figure 4). Such interlaced interactions add further evidence to the complex metabolic-cytokine relationships and mechanisms at play in the systemic host response to SARS-CoV-2 infection.

As a further complication, both quinolinic acid and kynurenic acid are known to modulate glutamic acid function, with increases in quinolinic acid resulting in cellular glutamic acid release. ${ }^{65}$ A substantial glutamic acid release may also explain the significantly lower glutamine/glutamic acid ratio observed in the SARS-CoV-2 (+) group in these data. This relationship between glutamic acid and glutamine is also pivotal in the regulation of homeostasis in immune cells, including T-cells and astrocytes within the CNS, ${ }^{66}$ where it acts as an immunomodulator, with the upregulation of glutamate receptors on their surface upon their activation. ${ }^{67,68}$ The glutamine/glutamic ratio is often cited in support of the concept of "immunometabolism", with the rate of glutamine consumption increased by immune cells when placed under catabolic conditions such as sepsis. ${ }^{69}$

Finally, cluster F (Figure 6) contains indole-3-acetic acid and the amino acids tryptophan, histidine, and citrulline are detected at lower concentrations in the SARS-CoV-2 (+) group compared with the HC and SARS-CoV-2 (-) individuals. As previously discussed, tryptophan is catabolized to kynurenine via the enzyme indoleamine 2,3-dioxygenase (IDO) (Figure 3B)-an important enzyme thought to regulate immune response in the peripheral system ${ }^{48}$ and the antimicrobial activity of interferons. ${ }^{49,50}$ The majority of indole-3-acetic acid is reported to be a product of tryptophan catabolism by the gut microbiome; ${ }^{70}$ however, in vitro experiments have demonstrated endogenous mammalian production. $^{71}$ Indole-3-acetic acid has previously been associated with SARS-CoV-2 infection; ${ }^{55}$ however, its role in the host phenotypic response remains unclear. Histidine has been associated with inflammation and oxidative stress and has been reported to influence the inflammatory cascade and have anti-inflammatory and antisecretory properties. ${ }^{72}$ Histidine has been shown to inhibit oxidative stress and downregulate TNF- $\alpha$ and, in the present study, was shown to be negatively associated with TNF- $\alpha$. Arginine is an important initiator of immune response through catabolic production of nitric oxide (NO) by inducible nitric oxide synthases (iNOS) producing citrulline as a byproduct. Low plasma citrulline levels have previously been associated with acute respiratory distress syndrome in patients with severe sepsis. ${ }^{73}$ Decreased bioavailability in arginine and citrulline during inflammation can impede activation of T-lymphocytes and macrophage anti-inflammatory responses through reduced capacity to support iNOS activity in SARS-CoV-2 (+) patients. This may have implications for vascular function and endothelial cell function with reduced NO from immune cells attributing to immune suppression. ${ }^{74}$ Recently, Adusumilli and colleagues suggested NO for therapeutic use, as it has demonstrated promise in similar respiratory disease models. ${ }^{75}$ It is plausible that NO could improve pulmonary 
vascular function in SARS-CoV-2 infection, through the vasodilation of blood vessels and increasing the amount of blood flow to alveoli, which in turn may improve oxygenation.

\section{CONCLUSIONS}

Enhanced knowledge of the molecular mechanisms that underpin host-virus interactions in SARS-CoV-2 infection is critical to both clinical management and improved diagnostic tools that can stratify patients into risk categories or who require urgent intervention. These data support the concept of COVID-19-induced metabolic phenoconversion and demonstrate the importance of interrogating an individual's metabolic phenotype to elucidate the systemic impact of infection. The identification of complex metabolic and systemic relationships that associate with the host response to viral infection offer insights into specific pathways that are directly influenced during acute infection.

The longitudinal case study demonstrates a time course pattern of infection using the metabolic phenotype, suggesting that such measures could be critical in the evaluation of longterm health impact of the virus. In particular, the significant metabolic imbalance between those metabolites previously reported to be associated with neurotoxicity (quinolinic acid, 3-hydroxykynurenine) and those reported to act as neuroprotective (kynurenic acid) and neurotransmitter (glutamic acid) molecules observed in these data following SARS-CoV-2 infection warrants further investigation, as it may be a pathway of mechanistic significance where SARS-CoV-2 infection leads to secondary neurological complications. Furthermore, this may become of particular relevance as more research and details emerge around the ever-increasing numbers of "longCOVID" syndrome, where individuals experience debilitating symptoms many months post-infection and the possibility of using amino acid and other lipid and lipoprotein markers ${ }^{76,77}$ as a means of assessing functional recovery in long-term patients.

\section{ASSOCIATED CONTENT}

\section{SI Supporting Information}

The Supporting Information is available free of charge at https://pubs.acs.org/doi/10.1021/acs.jproteome.1c00052.

Materials and methods; quantification of amino acids; quantification of tryptophan pathway metabolites; patient demographics for each of the study groups (Table S1); univariate analysis of cytokine differences between healthy control $(n=10)$ and SARS-CoV-2 $(+)$ $(n=7)$ groups (Table S2); comparison of plasma metabolites between SARS-CoV-2/HC and SARS-CoV$2(+)$ groups that revealed no significant differences in univariate Mann-Whitney $U$ tests (Table 1) (Figure $\mathrm{S1}$ ); three group boxplot comparisons of the plasma metabolites that were present at significantly different concentrations in the Mann-Whitney univariate testing $(p<0.05)$ (Figure S2); three group boxplot comparisons of the plasma metabolites that were not present at significant different concentrations in the Mann-Whitney univariate testing $(p>0.05)$ (Figure S3); comparison of serum cytokines discriminating groups healthy vs SARS-CoV-2 (+); (Figure S4) (PDF)

\section{AUTHOR INFORMATION}

\section{Corresponding Authors}

Luke Whiley - Australian National Phenome Centre, Computational and Systems Medicine, Health Futures Institute, Murdoch University, Perth, WA 6150, Australia; Perron Institute for Neurological and Translational Science, Nedlands, WA 6009, Australia; 다이.org/0000-00029088-4799; Email: luke.whiley@murdoch.edu.au

Jeremy K. Nicholson - Australian National Phenome Centre, Computational and Systems Medicine, Health Futures Institute, Murdoch University, Perth, WA 6150, Australia; Medical School, Faculty of Health and Medical Sciences, University of Western Australia, Nedlands, WA 6009, Australia; Institute of Global Health Innovation, Imperial College London, London SW7 2AZ, U.K.; (1) orcid.org/ 0000-0002-8123-8349; Email: Jeremy.nicholson@ murdoch.edu.au, j.nicholson@imperial.ac.uk

\section{Authors}

Nathan G. Lawler - Australian National Phenome Centre, Computational and Systems Medicine, Health Futures Institute, Murdoch University, Perth, WA 6150, Australia; 다잉.org/0000-0001-9649-425X

Nicola Gray - Australian National Phenome Centre, Computational and Systems Medicine, Health Futures Institute, Murdoch University, Perth, WA 6150, Australia; (1) orcid.org/0000-0002-0094-5245

Torben Kimhofer - Australian National Phenome Centre, Computational and Systems Medicine, Health Futures Institute, Murdoch University, Perth, WA 6150, Australia; (-) orcid.org/0000-0001-7158-9930

Berin Boughton - Australian National Phenome Centre, Computational and Systems Medicine, Health Futures Institute, Murdoch University, Perth, WA 6150, Australia; (1) orcid.org/0000-0001-6342-9814

Melvin Gay - Bruker Pty Ltd., Preston, VIC 3072, Australia

Rongchang Yang - Australian National Phenome Centre, Computational and Systems Medicine, Health Futures Institute, Murdoch University, Perth, WA 6150, Australia

Aude-Claire Morillon - Australian National Phenome Centre, Computational and Systems Medicine, Health Futures Institute, Murdoch University, Perth, WA 6150, Australia

Sung-Tong Chin - Australian National Phenome Centre, Computational and Systems Medicine, Health Futures Institute, Murdoch University, Perth, WA 6150, Australia

Monique Ryan - Australian National Phenome Centre, Computational and Systems Medicine, Health Futures Institute, Murdoch University, Perth, WA 6150, Australia

Sofina Begum - Australian National Phenome Centre, Computational and Systems Medicine, Health Futures Institute, Murdoch University, Perth, WA 6150, Australia; Department of Metabolism Digestion and Reproduction, Faculty of Medicine, Imperial College London, London SW7 2AZ, U.K.

Sze How Bong - Australian National Phenome Centre, Computational and Systems Medicine, Health Futures Institute, Murdoch University, Perth, WA 6150, Australia; (1) orcid.org/0000-0002-3313-5097

Jerome D. Coudert - Centre for Molecular Medicine \& Innovative Therapeutics, Murdoch University, Perth, WA 6150, Australia 
Dale Edgar - State Adult Burn Unit, Fiona Stanley Hospital, Murdoch, WA 6150, Australia; Burn Injury Research Node, The University of Notre Dame, Fremantle, WA 6160, Australia; Fiona Wood Foundation, Murdoch, WA 6150, Australia

Edward Raby - Department of Microbiology, PathWest Laboratory Medicine, Perth, WA 6009, Australia; Department of Infectious Diseases, Fiona Stanley Hospital, Perth, WA 6150, Australia

Sven Pettersson - Singapore National Neuro Science Centre, Singapore 308232, Singapore; Lee Kong Chian School of Medicine, Nanyang Technological University, Singapore 308232, Singapore; Department of Life Science Centre, Sunway University, 55100 Kuala Lumpur, Malaysia

Toby Richards - Medical School, Faculty of Health and Medical Sciences, University of Western Australia, Nedlands, WA 6009, Australia

Elaine Holmes - Australian National Phenome Centre, Computational and Systems Medicine, Health Futures Institute, Murdoch University, Perth, WA 6150, Australia; Department of Metabolism Digestion and Reproduction, Faculty of Medicine, Imperial College London, London SW7 2AZ, U.K.; ○ orcid.org/0000-0002-0556-8389

Complete contact information is available at: https://pubs.acs.org/10.1021/acs.jproteome.1c00052

\section{Author Contributions}

${ }^{\circ}$ N.G.L. and N.G. contributed equally to this work.

Notes

The authors declare no competing financial interest.

\section{ACKNOWLEDGMENTS}

The authors thank Spinnaker Health Research Foundation, WA, The McCusker Foundation, WA, The Western Australian State Government, and the MRFF for funding the Australian National Phenome Centre for this and related work. They acknowledge the COVID-19 Research Response Clinical Data and Biobank Team (https://wahtn.org).

\section{REFERENCES}

(1) World Health Organization. https://covid19.who.int (accessed Feb 24, 2021).

(2) Zhao, Y.-m.; Shang, Y.-m.; Song, W.-b.; Li, Q.-q.; Xie, H.; Xu, Q.-f.; Jia, J.-1.; Li, L.-m.; Mao, H.-1.; Zhou, X.-m.; Luo, H.; Gao, Y.-f.; $\mathrm{Xu}$, A.-g. Follow-up Study of the Pulmonary Function and Related Physiological Characteristics of COVID-19 Survivors Three Months after Recovery. EClinicalMedicine 2020, 25, No. 100463.

(3) Bompard, F.; Monnier, H.; Saab, I.; Tordjman, M.; Abdoul, H.; Fournier, L.; Sanchez, O.; Lorut, C.; Chassagnon, G.; Revel, M. P. Pulmonary Embolism in Patients with COVID-19 Pneumonia. Eur. Respir. J. 2020, 56, No. 2001365.

(4) Zheng, Y. Y.; Ma, Y. T.; Zhang, J. Y.; Xie, X. COVID-19 and the Cardiovascular System. Nat. Rev. Cardiol. 2020, 17, 259-260.

(5) Cai, Q.; Huang, D.; Yu, H.; Zhu, Z.; Xia, Z.; Su, Y.; Li, Z.; Zhou, G.; Gou, J.; Qu, J.; Sun, Y.; Liu, Y.; He, Q.; Chen, J.; Liu, L.; $\mathrm{Xu}, \mathrm{L}$. COVID-19: Abnormal Liver Function Tests. J. Hepatol. 2020, 73, 566-574.

(6) Mao, R.; Qiu, Y.; He, J. S.; Tan, J. Y.; Li, X. H.; Liang, J.; Shen, J.; Zhu, L. R.; Chen, Y.; Iacucci, M.; Ng, S. C.; Ghosh, S.; Chen, M. H. Manifestations and Prognosis of Gastrointestinal and Liver Involvement in Patients with COVID-19: A Systematic Review and Meta-Analysis. Lancet Gastroenterol. Hepatol. 2020, 5, 667-678.
(7) Ronco, C.; Reis, T.; Husain-Syed, F. Management of Acute Kidney Injury in Patients with COVID-19. Lancet Respir. Med. 2020, $8,738-742$.

(8) Paterson, R. W.; Brown, R. L.; Benjamin, L.; Nortley, R.; Wiethoff, S.; Bharucha, T.; Jayaseelan, D. L.; Kumar, G.; Raftopoulos, R. E.; Zambreanu, L.; Vivekanandam, V.; Khoo, A.; Geraldes, R.; Chinthapalli, K.; Boyd, E.; Tuzlali, H.; Price, G.; Christofi, G.; Morrow, J.; McNamara, P.; McLoughlin, B.; Lim, S. T.; Mehta, P. R.; Levee, V.; Keddie, S.; Yong, W.; Trip, S. A.; Foulkes, A. J. M.; Hotton, G.; Miller, T. D.; Everitt, A. D.; Carswell, C.; Davies, N. W. S.; Yoong, M.; Attwell, D.; Sreedharan, J.; Silber, E.; Schott, J. M.; Chandratheva, A.; Perry, R. J.; Simister, R.; Checkley, A.; Longley, N.; Farmer, S. F.; Carletti, F.; Houlihan, C.; Thom, M.; Lunn, M. P.; Spillane, J.; Howard, R.; Vincent, A.; Werring, D. J.; Hoskote, C.; Jäger, H. R.; Manji, H.; Zandi, M. S. The Emerging Spectrum of COVID-19 Neurology: Clinical, Radiological and Laboratory Findings. Brain 2020, 143, 3104-3120.

(9) Ellul, M. A.; Benjamin, L.; Singh, B.; Lant, S.; Michael, B. D.; Easton, A.; Kneen, R.; Defres, S.; Sejvar, J.; Solomon, T. Neurological Associations of COVID-19. Lancet Neurol. 2020, 19, 767-783.

(10) Helms, J.; Kremer, S.; Merdji, H.; Clere-Jehl, R.; Schenck, M.; Kummerlen, C.; Collange, O.; Boulay, C.; Fafi-Kremer, S.; Ohana, M.; Anheim, M.; Meziani, F. Neurologic Features in Severe SARSCoV-2 Infection. N. Engl. J. Med. 2020, 382, 2268-2270.

(11) Chee, Y. J.; Ng, S. J. H.; Yeoh, E. Diabetic Ketoacidosis Precipitated by Covid-19 in a Patient with Newly Diagnosed Diabetes Mellitus. Diabetes Res. Clin. Pract. 2020, 164, No. 108166.

(12) Li, J.; Wang, X.; Chen, J.; Zuo, X.; Zhang, H.; Deng, A. COVID-19 Infection May Cause Ketosis and Ketoacidosis. Diabetes, Obes. Metab. 2020, 22, 1935-1941.

(13) Ren, H.; Yang, Y.; Wang, F.; Yan, Y.; Shi, X.; Dong, K.; Yu, X.; Zhang, S. Association of the Insulin Resistance Marker TyG Index with the Severity and Mortality of COVID-19. Cardiovasc. Diabetol. 2020, 19, No. 58.

(14) Oxley, T. J.; Mocco, J.; Majidi, S.; Kellner, C. P.; Shoirah, H.; Singh, I. P.; De Leacy, R. A.; Shigematsu, T.; Ladner, T. R.; Yaeger, K. A.; Skliut, M.; Weinberger, J.; Dangayach, N. S.; Bederson, J. B.; Tuhrim, S.; Fifi, J. T. Large-Vessel Stroke as a Presenting Feature of Covid-19 in the Young. N. Engl. J. Med. 2020, 382, No. e60.

(15) Mahase, E. Covid-19: What Do We Know about "Long Covid"? BMJ 2020, 370, No. m2815.

(16) Lindon, J. C.; Nicholson, J. K. The Emergent Role of Metabolic Phenotyping in Dynamic Patient Stratification. Expert Opin. Drug Metab. Toxicol. 2014, 10, 915-919.

(17) Nicholson, J. K.; Holmes, E.; Kinross, J. M.; Darzi, A. W.; Takats, Z.; Lindon, J. C. Metabolic Phenotyping in Clinical and Surgical Environments. Nature 2012, 491, 384-392.

(18) Shen, B.; Yi, X.; Sun, Y.; Bi, X.; Du, J.; Zhang, C.; Quan, S.; Zhang, F.; Sun, R.; Qian, L.; Ge, W.; Liu, W.; Liang, S.; Chen, H.; Zhang, Y.; Li, J.; Xu, J.; He, Z.; Chen, B.; Wang, J.; Yan, H.; Zheng, Y.; Wang, D.; Zhu, J.; Kong, Z.; Kang, Z.; Liang, X.; Ding, X.; Ruan, G.; Xiang, N.; Cai, X.; Gao, H.; Li, L.; Li, S.; Xiao, Q.; Lu, T.; Zhu, Y.; Liu, H.; Chen, H.; Guo, T. Proteomic and Metabolomic Characterization of COVID-19 Patient Sera. Cell 2020, 182, 59.e1572.e15.

(19) Wu, D.; Shu, T.; Yang, X.; Song, J.-X. X.; Zhang, M.; Yao, C.; Liu, W.; Huang, M.; Yu, Y.; Yang, Q.; Zhu, T.; Xu, J.; Mu, J.; Wang, Y.; Wang, H.; Tang, T.; Ren, Y.; Wu, Y.; Lin, S.-H. H.; Qiu, Y.; Zhang, D.-Y. Y.; Shang, Y.; Zhou, X. Plasma Metabolomic and Lipidomic Alterations Associated with COVID-19. Natl. Sci. Rev. 2020, 1157.

(20) Thomas, T.; Stefanoni, D.; Reisz, J. A.; Nemkov, T.; Bertolone, L.; Francis, R. O.; Hudson, K. E.; Zimring, J. C.; Hansen, K. C.; Hod, E. A.; Spitalnik, S. L.; D’Alessandro, A. COVID-19 Infection Alters Kynurenine and Fatty Acid Metabolism, Correlating with IL-6 Levels and Renal Status. JCI Insight 2020, 5, No. e140327.

(21) Kimhofer, T.; Lodge, S.; Whiley, L.; Gray, N.; Loo, R. L.; Lawler, N. G.; Nitschke, P.; Bong, S. H.; Morrison, D. L.; Begum, S.; Richards, T.; Yeap, B. B.; Smith, C.; Smith, K. G. C.; Holmes, E.; 
Nicholson, J. K. Integrative Modeling of Quantitative Plasma Lipoprotein, Metabolic, and Amino Acid Data Reveals a Multiorgan Pathological Signature of SARS-CoV-2 Infection. J. Proteome Res. 2020, 19, 4442-4454.

(22) Wu, Q.; Zhou, L.; Sun, X.; Yan, Z.; Hu, C.; Wu, J.; Xu, L.; Li, X.; Liu, H.; Yin, P.; Li, K.; Zhao, J.; Li, Y.; Wang, X.; Li, Y.; Zhang, Q.; Xu, G.; Chen, H. Altered Lipid Metabolism in Recovered SARS Patients Twelve Years after Infection. Sci. Rep. 2017, 7, No. 9110.

(23) Chan, H. L. Y.; Leung, W. K.; To, K. F.; Chan, P. K. S.; Lee, N.; Wu, A.; Tam, J. S. L.; Sung, J. J. Y. Retrospective Analysis of Liver Function Derangement in Severe Acute Respiratory Syndrome. Am. J. Med. 2004, 116, 566-567.

(24) Yan, B.; Chu, H.; Yang, D.; Sze, K. H.; Lai, P. M.; Yuan, S.; Shuai, H.; Wang, Y.; Kao, R. Y. T.; Chan, J. F. W.; Yuen, K. Y. Characterization of the Lipidomic Profile of Human CoronavirusInfected Cells: Implications for Lipid Metabolism Remodeling upon Coronavirus Replication. Viruses 2019, 11, No. 73.

(25) CRR. COVID Research Response Collaboration, 2021. https://research-au.net/covid-research-response/ (last accessed Feb 23, 2021).

(26) Darnell, M. E. R.; Subbarao, K.; Feinstone, S. M.; Taylor, D. R. Inactivation of the Coronavirus That Induces Severe Acute Respiratory Syndrome, SARS-CoV. J. Virol. Methods 2004, 121, 85-91.

(27) Loo, R. L.; Lodge, S.; Kimhofer, T.; Bong, S. H.; Begum, S.; Whiley, L.; Gray, N.; Lindon, J. C.; Nitschke, P.; Lawler, N. G.; Schäfer, H.; Spraul, M.; Richards, T.; Nicholson, J. K.; Holmes, E. Quantitative In-Vitro Diagnostic NMR Spectroscopy for Lipoprotein and Metabolite Measurements in Plasma and Serum: Recommendations for Analytical Artifact Minimization with Special Reference to COVID-19/SARS-CoV-2 Samples. J. Proteome Res. 2020, 19, 44284441.

(28) Whiley, L.; Nye, L. C.; Grant, I.; Andreas, N.; Chappell, K. E.; Sarafian, M. H.; Misra, R.; Plumb, R. S.; Lewis, M. R.; Nicholson, J. K.; Holmes, E.; Swann, J. R.; Wilson, I. D. Ultrahigh-Performance Liquid Chromatography Tandem Mass Spectrometry with Electrospray Ionization Quantification of Tryptophan Metabolites and Markers of Gut Health in Serum and Plasma - Application to Clinical and Epidemiology Cohorts. Anal. Chem. 2019, 91, 5207-5216.

(29) Gray, N.; Zia, R.; King, A.; Patel, V. C.; Wendon, J.; McPhail, M. J. W.; Coen, M.; Plumb, R. S.; Wilson, I. D.; Nicholson, J. K. High-Speed Quantitative UPLC-MS Analysis of Multiple Amines in Human Plasma and Serum via Precolumn Derivatization with 6Aminoquinolyl-N-Hydroxysuccinimidyl Carbamate: Application to Acetaminophen-Induced Liver Failure. Anal. Chem. 2017, 89, 24782487.

(30) Gray, N.; Lawler, N. G.; Yang, R.; Morillon, A. C.; Gay, M. C. L.; Bong, S. H.; Holmes, E.; Nicholson, J. K.; Whiley, L. A Simultaneous Exploratory and Quantitative Amino Acid and Biogenic Amine Metabolic Profiling Platform for Rapid Disease Phenotyping via UPLC-QToF-MS. Talanta 2021, 223, No. 121872.

(31) Kimhofer, T. MetaboM8, 2020. Https://Github.Com/ Tkimhofer/ (last accessed Feb 23, 2021).

(32) Storey, J. D. The Positive False Discovery Rate: A Bayesian Interpretation and the q-Value. Ann. Stat. 2003, 31, 2013-2035.

(33) Benjamini, Y.; Hochberg, Y. Controlling the False Discovery Rate: A Practical and Powerful Approach to Multiple Testing. J. $R$. Stat. Soc., Ser. B 1995, 57, 289-300.

(34) Quist-Paulsen, E.; Aukrust, P.; Kran, A. M. B.; Dunlop, O.; Ormaasen, V.; Stiksrud, B.; Midttun, Ø.; Ueland, T.; Ueland, P. M.; Mollnes, T. E.; Dyrhol-Riise, A. M. High Neopterin and IP-10 Levels in Cerebrospinal Fluid Are Associated with Neurotoxic Tryptophan Metabolites in Acute Central Nervous System Infections 11 Medical and Health Sciences 1103 Clinical Sciences. J. Neuroinflammation 2018, 15, No. 327.

(35) Lugo-Huitrón, R.; Ugalde Muñiz, P.; Pineda, B.; PedrazaChaverrí, J.; Ríos, C.; Pérez-De La Cruz, V. Quinolinic Acid: An Endogenous Neurotoxin with Multiple Targets. Oxid. Med. Cell. Longevity 2013, 1-14.
(36) Guillemin, G. J. Quinolinic Acid, the Inescapable Neurotoxin. FEBS J. 2012, 279, 1356-1365.

(37) Sundaram, G.; Brew, B. J.; Jones, S. P.; Adams, S.; Lim, C. K.; Guillemin, G. J. Quinolinic Acid Toxicity on Oligodendroglial Cells: Relevance for Multiple Sclerosis and Therapeutic Strategies. J. Neuroinflammation 2014, 11, No. 204.

(38) Heilman, P. L.; Wang, E. W.; Lewis, M. M.; Krzyzanowski, S.; Capan, C. D.; Burmeister, A. R.; Du, G.; Escobar Galvis, M. L.; Brundin, P.; Huang, X.; Brundin, L. Tryptophan Metabolites Are Associated With Symptoms and Nigral Pathology in Parkinson's Disease. Mov. Disord. 2020, 35, 2028-2037.

(39) Johansson, A. S.; Owe-Larsson, B.; Asp, L.; Kocki, T.; Adler, M.; Hetta, J.; Gardner, R.; Lundkvist, G. B. S.; Urbanska, E. M.; Karlsson, H. Activation of Kynurenine Pathway in Exvivo Fibroblasts from Patients with Bipolar Disorder or Schizophrenia: Cytokine Challenge Increases Production of 3-Hydroxykynurenine. J. Psychiatr. Res. 2013, 47, 1815-1823.

(40) Okuda, S.; Nishiyama, N.; Saito, H.; Katsuki, H. 3Hydroxykynurenine, an Endogenous Oxidative Stress Generator, Causes Neuronal Cell Death with Apoptotic Features and Region Selectivity. J. Neurochem. 1998, 70, 299-307.

(41) Quist-Paulsen, E.; Ormaasen, V.; Kran, A. M. B.; Dunlop, O.; Ueland, P. M.; Ueland, T.; Eikeland, R.; Aukrust, P.; Nordenmark, T. H. Encephalitis and Aseptic Meningitis: Short-Term and Long-Term Outcome, Quality of Life and Neuropsychological Functioning. Sci. Rep. 2019, 9, No. 16158.

(42) Wu, W.; Nicolazzo, J. A.; Wen, L.; Chung, R.; Stankovic, R.; Bao, S. S.; Lim, C. K.; Brew, B. J.; Cullen, K. M.; Guillemin, G. J. Expression of Tryptophan 2,3-Dioxygenase and Production of Kynurenine Pathway Metabolites in Triple Transgenic Mice and Human Alzheimer's Disease Brain. PLoS One 2013, 8, No. e59749.

(43) Vécsei, L.; Beal, M. F. Huntington's Disease, Behavioral Disturbances, and Kynurenines: Preclinical Findings and Therapeutic Perspectives. Biol. Psychiatry 1996, 39, 1061-1063.

(44) Doty, R. L. Olfaction in Parkinson's Disease and Related Disorders. Neurobiol. Dis. 2012, 46, 527-552.

(45) Townsend, L.; Dyer, A. H.; Jones, K.; Dunne, J.; Mooney, A.; Gaffney, F.; O'Connor, L.; Leavy, D.; O’Brien, K.; Dowds, J.; Sugrue, J. A.; Hopkins, D.; Martin-Loeches, I.; Ni Cheallaigh, C.; Nadarajan, P.; McLaughlin, A. M.; Bourke, N. M.; Bergin, C.; O’Farrelly, C.; Bannan, C.; Conlon, N. Persistent Fatigue Following SARS-CoV-2 Infection Is Common and Independent of Severity of Initial Infection. PLoS One 2020, 15, No. e0240784.

(46) Åkesson, K.; Pettersson, S.; Ståhl, S.; Surowiec, I.; Hedenström, M.; Eketjäll, S.; Trygg, J.; Jakobsson, P. J.; Gunnarsson, I.; Svenungsson, E.; Idborg, H. Kynurenine Pathway Is Altered in Patients with SLE and Associated with Severe Fatigue. Lupus Sci. Med. 2018, 5, No. e000254.

(47) Conductier, G.; Blondeau, N.; Guyon, A.; Nahon, J. L.; Rovère, C. The Role of Monocyte Chemoattractant Protein MCP1/ CCL2 in Neuroinflammatory Diseases. J. Neuroimmunol. 2010, 224, 93-100.

(48) Mellor, A. L.; Munn, D. H. IDO Expression by Dendritic Cells: Tolerance and Tryptophan Catabolism. Nat. Rev. Immunol. 2004, 4, 762-774.

(49) Heseler, K.; Spekker, K.; Schmidt, S. K.; MacKenzie, C. R.; Däubener, W. Antimicrobial and Immunoregulatory Effects Mediated by Human Lung Cells: Role of IFN- $\gamma$-Induced Tryptophan Degradation. FEMS Immunol. Med. Microbiol. 2008, 52, 273-281.

(50) Murray, H. W.; Szuro-Sudol, A.; Wellner, D.; Oca, M. J.; Granger, A. M.; Libby, D. M.; Rothermel, C. D.; Rubin, B. Y. Role of Tryptophan Degradation in Respiratory Burst-Independent Antimicrobial Activity of Gamma Interferon-Stimulated Human Macrophages. Infect. Immun. 1989, 57, 845-849.

(51) Heyes, M. P. Relationship Between Interferon- $\gamma$, Indoleamine2,3-dioxygenase and Tryptophan. FASEB J. 1991, 5, 3003-3004.

(52) Sarkar, S. A.; Wong, R.; Hackl, S. I.; Moua, O.; Gill, R. G.; Wiseman, A.; Davidson, H. W.; Hutton, J. C. Induction of 
Indoleamine 2,3-Dioxygenase by Interferon- $\gamma$ in Human Islets. Diabetes 2007, 56, 72-79.

(53) Huber, C.; Richard Batchelor, J.; Fuchs, D.; Hausen, A.; Lang, A.; Niederwieser, D.; Reibnegger, G.; Swetly, P.; Troppmair, J.; Wachter, H. Immune Response-Associated Production of Neopterin: Release from Macrophages Primarily under Control of InterferonGamma. J. Exp. Med. 1984, 160, 310-316.

(54) Digby, J. E.; Martinez, F.; Jefferson, A.; Ruparelia, N.; Chai, J.; Wamil, M.; Greaves, D. R.; Choudhury, R. P. Anti-Inflammatory Effects of Nicotinic Acid in Human Monocytes Are Mediated by GPR109A Dependent Mechanisms. Arterioscler., Thromb., Vasc. Biol. 2012, 32, 669-676.

(55) Blasco, H.; Bessy, C.; Plantier, L.; Lefevre, A.; Piver, E.; Bernard, L.; Marlet, J.; Stefic, K.; Benz-de Bretagne, I.; Cannet, P.; Lumbu, H.; Morel, T.; Boulard, P.; Andres, C. R.; Vourc'h, P.; Hérault, O.; Guillon, A.; Emond, P. The Specific Metabolome Profiling of Patients Infected by SARS-COV-2 Supports the Key Role of Tryptophan-Nicotinamide Pathway and Cytosine Metabolism. Sci. Rep. 2020, 10, No. 16824.

(56) Carlson, L. A. Nicotinic Acid: The Broad-Spectrum Lipid Drug. A 50th Anniversary Review. J. Intern. Med. 2005, 258, 94-114.

(57) Bruzzone, C.; Bizkarguenaga, M.; Gil-Redondo, R.; Diercks, T.; Arana, E.; García de Vicuña, A.; Seco, M.; Bosch, A.; Palazón, A.; San Juan, I.; Laín, A.; Gil-Martínez, J.; Bernardo-Seisdedos, G.; Fernández-Ramos, D.; Lopitz-Otsoa, F.; Embade, N.; Lu, S.; Mato, J. M.; Millet, O. SARS-CoV-2 Infection Dysregulates the Metabolomic and Lipidomic Profiles of Serum. iScience 2020, 23, No. 101645.

(58) Chang, M. L.; Yang, S. S. Metabolic Signature of Hepatic Fibrosis: From Individual Pathways to Systems Biology. Cells 2019, 8, No. 1423.

(59) Nakamura, Y.; Mizuguchi, T.; Kawamoto, M.; Meguro, M.; Harada, K.; Ota, S.; Hirata, K. Cluster Analysis of Indicators of Liver Functional and Preoperative Low Branched-Chain Amino Acid Tyrosine Ration Indicate a High Risk of Early Recurrence in Analysis of 165 Hepatocellular Carcinoma Patients after Initial Hepatectomy. Surgery 2011, 150, 250-262.

(60) Rosen, H. M.; Yoshimura, N.; Hodgman, J. M.; Fischer, J. E. Plasma Amino Acid Patterns in Hepatic Encephalopathy of Differing Etiology. Gastroenterology 1977, 72, 483-487.

(61) Fischer, J. E.; Rosen, H. M.; Ebeid, A. M.; James, J. H.; Keane, J. M.; Soeters, P. B. The Effect of Normalization of Plasma Amino Acids on Hepatic Encephalopathy in Man. Surgery 1976, 80, 77-91.

(62) Beckwith-Hall, B. M.; Nicholson, J. K.; Nicholls, A. W.; Foxall, P. J. D.; Lindon, J. C.; Connor, S. C.; Abdi, M.; Connelly, J.; Holmes, E. Nuclear Magnetic Resonance Spectroscopic and Principal Components Analysis Investigations into Biochemical Effects of Three Model Hepatotoxins. Chem. Res. Toxicol. 1998, 11, 260-272.

(63) Bhatnagar, S. K.; Welty, J. D.; Al Yusuf, A. R. Significance of Blood Taurine Levels in Patients with First Time Acute Ischaemic Cardiac Pain. Int. J. Cardiol. 1990, 27, 361-366.

(64) Geisler, S.; Gostner, J. M.; Becker, K.; Ueberall, F.; Fuchs, D. Immune Activation and Inflammation Increase the Plasma Phenylalanine-to- Tyrosine Ratio. Pteridines 2013, 24, 27-31.

(65) Schwarcz, R. Kynurenines and Glutamate. Multiple Links and Therapeutic Implications. Adv. Pharmacol. 2016, 76, 13-37.

(66) Schousboe, A.; Waagepetersen, H. S. Role of Astrocytes in Glutamate Homeostasis: Implications for Excitotoxicity. Neurotoxic. Res. 2005, 8, 221-225.

(67) Shanker, A.; de Aquino, M. T. P.; Hodo, T.; Uzhachenko, R. Glutamate Receptors Provide Costimulatory Signals to Improve T Cell Immune Response. J. Immunol. 2018, 200, 47.24.

(68) Pacheco, R.; Gallart, T.; Lluis, C.; Franco, R. Role of Glutamate on T-Cell Mediated Immunity. J. Neuroimmunol. 2007, 185, 9-19.

(69) Newsholme, P. Why Is L-Glutamine Metabolism Important to Cells of the Immune System in Health, Postinjury, Surgery or Infection? J. Nutr. 2001, 131, $2515 S$.
(70) Smith, E. A.; Macfarlane, G. T. Formation of Phenolic and Indolic Compounds by Anaerobic Bacteria in the Human Large Intestine. Microb. Ecol. 1997, 33, 180-188.

(71) Zhang, X.; Gan, M.; Li, J.; Li, H.; Su, M.; Tan, D.; Wang, S.; Jia, M.; Zhang, L.; Chen, G. Endogenous Indole Pyruvate Pathway for Tryptophan Metabolism Mediated by IL4I1. J. Agric. Food Chem. 2020, 68, 10678-10684.

(72) Watanabe, M.; Suliman, M. E.; Qureshi, A. R.; Garcia-Lopez, E.; Bárány, P.; Heimbürger, O.; Stenvinkel, P.; Lindholm, B. Consequences of Low Plasma Histidine in Chronic Kidney Disease Patients: Associations with Inflammation, Oxidative Stress, and Mortality. Am. J. Clin. Nutr. 2008, 87, 1860-1866.

(73) Ware, L. B.; Magarik, J. A.; Wickersham, N.; Cunningham, G.; Rice, T. W.; Christman, B. W.; Wheeler, A. P.; Bernard, G. R.; Summar, M. L. Low Plasma Citrulline Levels Are Associated with Acute Respiratory Distress Syndrome in Patients with Severe Sepsis. Crit. Care 2013, 17, R10.

(74) Wijnands, K. A. P.; Castermans, T. M. R.; Hommen, M. P. J.; Meesters, D. M.; Poeze, M. Arginine and Citrulline and the Immune Response in Sepsis. Nutrients 2015, 7, 1426-1463.

(75) Adusumilli, N. C.; Zhang, D.; Friedman, J. M.; Friedman, A. J. Harnessing Nitric Oxide for Preventing, Limiting and Treating the Severe Pulmonary Consequences of COVID-19. Nitric Oxide 2020, 103, 4-8.

(76) Lodge, S.; Nitschke, P.; Kimhofer, T.; Coudert, J. D.; Begum, S.; Bong, S. H.; Richards, T.; Edgar, D.; Raby, E.; Spraul, M.; Schaefer, H.; Lindon, J. C.; Loo, R. L.; Holmes, E.; Nicholson, J. K. NMR Spectroscopic Windows on the Systemic Effects of SARS-CoV2 Infection on Plasma Lipoproteins and Metabolites in Relation to Circulating Cytokines. J. Proteome Res. 2021, 1382.

(77) Lodge, S.; Nitschke, P.; Kimhofer, T.; Wist, J.; Bong, S.-H.; Loo, R. L.; Masuda, R.; Begum, S.; Richards, T.; Lindon, J. C.; Bermel, W.; Reinsperger, T.; Schaefer, H.; Spraul, M.; Holmes, E.; Nicholson, J. K. Diffusion and Relaxation Edited Proton NMR Spectroscopy of Plasma Reveals a High-Fidelity Supramolecular Biomarker Signature of SARS-CoV-2 Infection. Anal. Chem. 2021, 3976. 